\title{
Escherichia coli rimM and yjeQ null strains accumulate immature 30S subunits of similar structure and protein complement
}

\author{
VIVIAN LEONG, MEREDITH KENT, AHMAD JOMAA, and JOAQUIN ORTEGA ${ }^{1}$ \\ Department of Biochemistry and Biomedical Sciences, and M.G. DeGroote Institute for Infectious Diseases Research, McMaster University, \\ Hamilton, Ontario, L8N3Z5, Canada
}

\begin{abstract}
Assembly of the Escherichia coli 30S ribosomal subunits proceeds through multiple parallel pathways. The protein factors RimM, YjeQ, RbfA, and Era work in conjunction to assist at the late stages of the maturation process of the small subunit. However, it is unclear how the functional interplay between these factors occurs in the context of multiple parallel pathways. To understand how these factors work together, we have characterized the immature $30 \mathrm{~S}$ subunits that accumulate in $\Delta$ rim $M$ cells and compared them with immature $30 \mathrm{~S}$ subunits from a $\Delta y j e Q$ strain. The cryo-EM maps obtained from these particles showed that the densities representing helices 44 and 45 in the rRNA were partially missing, suggesting mobility of these motifs. These 30S subunits were also partially depleted in all tertiary ribosomal proteins, particularly those binding in the head domain. Using image classification, we identified four subpopulations of $\Delta$ rim $M$ immature $30 \mathrm{~S}$ subunits differing in the amount of missing density for helices 44 and 45 , as well as the amount of density existing in these maps for the underrepresented proteins. The structural defects found in these immature subunits resembled those of the $30 \mathrm{~S}$ subunits that accumulate in the $\Delta y j \mathrm{e} Q$ strain. These findings are consistent with an "early convergency model" in which multiple parallel assembly pathways of the 30S subunit converge into a late assembly intermediate, as opposed to the mature state. Functionally related factors will bind to this intermediate to catalyze the last steps of maturation leading to the mature $30 \mathrm{~S}$ subunit.
\end{abstract}

Keywords: ribosome assembly; $30 S$ subunit; RimM protein; YjeQ protein; cryo-electron microscopy

\section{INTRODUCTION}

The ribosome in Escherichia coli comprises three strands of RNA (16S, 23S, and 5S rRNA) and over 50 ribosomal proteins ( $r$-proteins). At least 20 protein factors facilitate the assembly of these components in vivo into the 70S ribosomal particle (Wilson and Nierhaus 2007). The exact role of most of these factors during the assembly of the ribosome is still unclear. Existing data suggest that one of the roles played by these cofactors is to assist the folding of the rRNA and allow the assembly process to proceed more quickly by preventing the large molecules of rRNA from falling into kinetic traps (Wilson and Nierhaus 2007; Britton 2009; Connolly and Culver 2009; Shajani et al. 2011).

Recent time-resolved hydroxyl radical footprinting studies in the bacterial 30S ribosomal subunit (Adilakshmi et al. 2008) suggest that the late stages of assembly of the particle, including the folding of the $3^{\prime}$ domain and decoding center, have a stronger tendency to fall into kinetic traps.

\footnotetext{
${ }^{1}$ Corresponding author

E-mail ortegaj@mcmaster.ca

Article published online ahead of print. Article and publication date are at http://www.rnajournal.org/cgi/doi/10.1261/rna.037523.112.
}

Accordingly, most of the described cofactors assisting assembly in the small ribosomal subunit are believed to act late on the maturation process. One of these factors is the RimM protein.

Bacterial strains lacking RimM show a sevenfold reduction in their growth rate and a decreased translation efficiency (Bylund et al. 1997). These strains also exhibit accumulation of free $30 \mathrm{~S}$ and $50 \mathrm{~S}$ subunits and fewer polysomes (Lovgren et al. 2004). Primer extension analysis has shown that strains lacking RimM accumulate immature 16S rRNA still containing the $115 \mathrm{nt}$ of the $5^{\prime}$ precursor sequence (Bylund et al. 1998). RimM associates with free 30S subunits, but it does not associate with $50 \mathrm{~S}$ subunits or $70 \mathrm{~S}$ particles (Bylund et al. 1997). The mechanism through which RimM facilitates the assembly of the $30 \mathrm{~S}$ subunit has remained elusive.

RimM is a $21-\mathrm{kDa}$ protein conserved in most eubacteria, but it is not found in Archaea, and it has been reported in only four eukaryotic species (Lovgren et al. 2004). RimM contains a characteristic N-terminal and a PRC (photosynthetic reaction center)-barrel C-terminal domain, linked by an unstructured region (Suzuki et al. 2007). The N-terminal domain comprises the first 80 residues of the protein, which fold into a closed $\beta$-barrel structure. The highly conserved 
GXXG motif contained in this region is part of a $\mathrm{KH}$ domain in other proteins, and it is often involved in mediating interactions with RNA (Grishin 2001). The PRC-barrel domain contains approximately the $75 \mathrm{C}$-terminal residues of RimM, and it is named after the $\mathrm{H}$ subunit of the photosynthetic reaction center in purple bacteria because of its structural similarities (Anantharaman and Aravind 2002). The $\mathrm{H}$ subunit of the photosynthetic reaction center in purple bacteria mediates electron transfer during photosynthesis. However, in RimM, the PRC-barrel domain lacks a conserved acidic residue that acts as a redox regulator during photosynthesis. Instead, this domain in RimM is believed to mediate the interaction with the rRNA or r-proteins of the 30S subunit. In this regard, a crystal structure of Thermus thermophilus RimM bound to S19 was deposited in the Protein Data Bank (PDB ID: 3A1P) and shows that the PRC-barrel forms multiple binding contacts with a $\beta$-sheet region of the S19 globular domain as well as with its extended C-terminal tail. The conserved tyrosine residues in position 106 and 107 located in the linker region between the two domains of RimM have also been found to be essential for the interaction with r-protein S19 (Lovgren and Wikstrom 2001; Lovgren et al. 2004).

Extensive genetic, structural, and biochemical evidence suggests that RimM assists in the late stages of assembly of the $30 \mathrm{~S}$ subunit in conjunction with at least three other factors: Era, RbfA, and YjeQ (also called RsgA) (Bylund et al. 1998, 2001; Inoue et al. 2003, 2006; Campbell and Brown 2008). Structurally, it has been shown that YjeQ, RbfA, and Era all bind in close proximity to the decoding center of the mature 30S subunit (Sharma et al. 2005; Datta et al. 2007; Guo et al. 2011; Jomaa et al. 2011b), suggesting that these factors play a joint role in the organization of the functional core of the small subunit. Genetic evidence suggests that RimM binds to a distinct region of the head of the $30 \mathrm{~S}$ subunit comprised of helices 31 and $33 \mathrm{~b}$ of the $16 \mathrm{~S}$ rRNA and r-proteins S13 and S19 right above the decoding center (Lovgren et al. 2004). With the PRC $\beta$-barrel domain of RimM bound to $\mathrm{S} 19$, it is plausible that the $\mathrm{N}$-terminal domain of the assembly factor may extend into the A site contacting RbfA or YjeQ to also mediate in the maturation of the decoding center. However, very little is known of how RimM may fit into a coordinated sequence of events, along with YjeQ, Era, and RbfA, to mediate this process.

To improve our understanding of the role of RimM in the late stages of assembly of the 30S subunit and its integration into a functional interplay with RbfA, Era, and YjeQ, we biochemically and structurally characterized the $30 \mathrm{~S}$ subunits that accumulate in a rimM null strain of $E$. coli. These ribosomal subunits accumulate unprocessed 16S rRNA and had an incomplete protein complement. In particular, all tertiary r-proteins showed some degree of depletion but mainly those located in the head domain. Cryo-electron microscopy (cryoEM) maps obtained from these particles showed missing densities for helices 44 and 45 of their rRNA and decreased densities for the depleted r-proteins. The conformational defects observed in the rRNA were incompatible with binding to the 50S subunits or participation in translation. More importantly, we found multiple similarities between these immature 30S subunits and those that accumulate in yjeQ null strains (Jomaa et al. 2011a). This finding will be discussed in the context of potential models framing the functional interplay of RimM, RbfA, Era, and YjeQ during the late stages of assembly of the $30 \mathrm{~S}$ subunit.

\section{RESULTS}

\section{Slow-growth phenotype and altered ribosome profiles in the rimM null strain from the Keio collection}

The purpose of this work was to characterize the immature 30 S subunits that accumulate in a $\Delta$ rimM strain of E. coli and compare them with the small ribosomal subunits that were previously isolated from a $\Delta y j e Q$ strain (Jomaa et al. 2011a). To eliminate differences caused by a diverse genotypic background, we performed our analysis with the same $E$. coli strain that was used previously in the $\Delta y j e Q$ study. In this case, the strain was harboring a clean deletion of rimM instead (referred to as $\triangle$ rimM strain hereafter). Both the $\Delta$ rim $M$ and $\triangle y j e Q$ strains used in these two studies are part of the Keio collection (Baba et al. 2006), which is a library of E. coli K12 strains with single-gene deletions of all nonessential genes. In each deletion, the open reading frame coding region was replaced by a kanamycin resistance cassette.

We used polymerase chain reaction (PCR) analysis to verify the deletion of the rimM gene in the E. coli strain and the presence of the kanamycin resistance cassette (Fig. 1A). The analysis revealed that the rimM gene was present in the parental strain (BW25113), but it had been removed in the $\Delta$ rim $M$ strain, which now contained the kanamycin resistance cassette.

It has been previously reported that that bacterial rim $M$ null strains of $E$. coli display a slow growth phenotype (Bylund et al. 1997, 1998). To determine whether the $\Delta$ rimM strain from the Keio collection had a similar phenotype, we compared the growth of the $\Delta$ rimM and parental strains at $37^{\circ} \mathrm{C}$ and $25^{\circ} \mathrm{C}$ in LB liquid and LB agar media. Growth profiles in the liquid media collected at slightly over twelve hours (Fig. 1B) showed that, at $37^{\circ} \mathrm{C}$, the doubling time at exponential phase of the $\Delta$ rim $M$ strain is 2.5 times higher, but it reaches almost a similar $\mathrm{OD}_{600}$ value at stationary phase as the parental strain. At $25^{\circ} \mathrm{C}$, the difference in doubling time between parental and $\Delta$ rim $M$ strain was similar (Table 1), but in this case, the density reached by the mutant strain was at least one order of magnitude lower than in the parental strain (Fig. 1B). The slower growth rate of the $\Delta$ rimM strain at $37^{\circ} \mathrm{C}$ and $25^{\circ} \mathrm{C}$ was also evident in solid media with the null strain showing colonies significantly smaller in size (Fig. 1C). These experiments allowed us to analyze whether the $\Delta$ rim $M$ strain from the Keio collection exhibited a cold- 
sensitive phenotype, which is a well-known hallmark of ribosome assembly defects in bacteria (Bylund et al. 1997, 1998; Campbell et al. 2006; Connolly and Culver 2009; Nord et al. 2009). However, we found that the ratios between the growth rates at $\log$ phase in the parental and $\Delta$ rimM strains at $37^{\circ} \mathrm{C}$ and $25^{\circ} \mathrm{C}$ were very similar (2.5 and 2.3 , respectively) (Table 1). Therefore, we concluded that a cold-sensitive phenotype in this strain was not apparent.

To ensure that the deletion of the rimM gene was responsible for the slow growth phenotype in the $\Delta$ rimM cells, the gene was reintroduced with the high-copy pCA24N plasmid. Reintroduction of the gene in a plasmid instead of in the chromosome eliminated the possibility of polar effects on downstream genes. In this vector, RimM expression is under the control of an isopropyl-beta-D-thiogalactopyranoside (IPTG)-inducible T5 promotor (Kitagawa et al. 2005). We found that, even in the absence of inducer, the leak in RimM expression was sufficient to partially correct the slow-growth phenotype of the $\Delta$ rimM strain (Fig. 1B,C). Indeed, overexpression of RimM protein by addition of IPTG to the media led to levels of protein that were toxic to the cells, preventing the recovery of the slow growth phenotype (data not shown). Transformation of the empty pCA24N vector in $\Delta$ rim $M$ cells had no effect on their growth (Fig. 1B, C) allowing us to conclude that the slow growth phenotype observed for this strain of the Keio collection was due to the absence of the RimM protein.

Previous work has also shown that rimM null strains exhibit accumulation of free $30 \mathrm{~S}$ and $50 \mathrm{~S}$ subunits and fewer polysomes (Lovgren et al. 2004). To assess the status of ribosomes in the $\Delta$ rimM strain from the Keio collection, we obtained ribosome (Fig. 1D) and polysome (Fig. 1E) profiles for this and the parental strains using sucrose gradient density centrifugation. Consistent with other null rimM strains, the $\Delta$ rimM strain from the Keio collection also showed a severe reduction of the proportion of $70 \mathrm{~S}$ ribosomes and a corresponding increase in the levels of free $30 \mathrm{~S}$ and $50 \mathrm{~S}$ subunits when compared to the parental strain. The percentage of $30 \mathrm{~S}$ subunits that were not associated into $70 \mathrm{~S}$ ribosomes in the $\Delta$ rimM strain was $\sim 47 \%$. In contrast, only $7 \%$ of the $30 \mathrm{~S}$ subunits were found in the free form in the parental strain (Fig. 1D). In addition, the polysome profile obtained from the $\Delta$ rim $M$ strain showed only a few polysomes, compared to the multiple and higher polysome peaks found in the parental strain (Fig. 1E).

\section{Immature 30S subunits with unprocessed rRNA and incomplete protein complement accumulate in the rimM null strain from the Keio collection}

In E. coli, the rRNA molecule for the $30 \mathrm{~S}$ subunit (16S rRNA) is synthesized as part of one primary transcript that includes as well the two rRNA molecules for the 50S subunit (23S and $5 \mathrm{~S}$ rRNAs). RNase III cleaves this primary transcript into three precursor rRNAs, each containing the sequence for the mature rRNA molecule plus additional precursor sequences at the $5^{\prime}$ and $3^{\prime}$ ends (Srivastava and Schlessinger 1990). The $17 \mathrm{~S}$ rRNA is the precursor rRNA for the $30 \mathrm{~S}$ subunit and contains 115 and 33 additional nucleotides in its $5^{\prime}$ and $3^{\prime}$ ends, respectively. These precursor sequences are cleaved by RNase E and RNase G ( $5^{\prime}$ end) and a still uncharacterized RNase ( $3^{\prime}$ end) producing the $16 \mathrm{~S}$ rRNA that is present in mature $30 \mathrm{~S}$ subunits (Li et al. 1999).
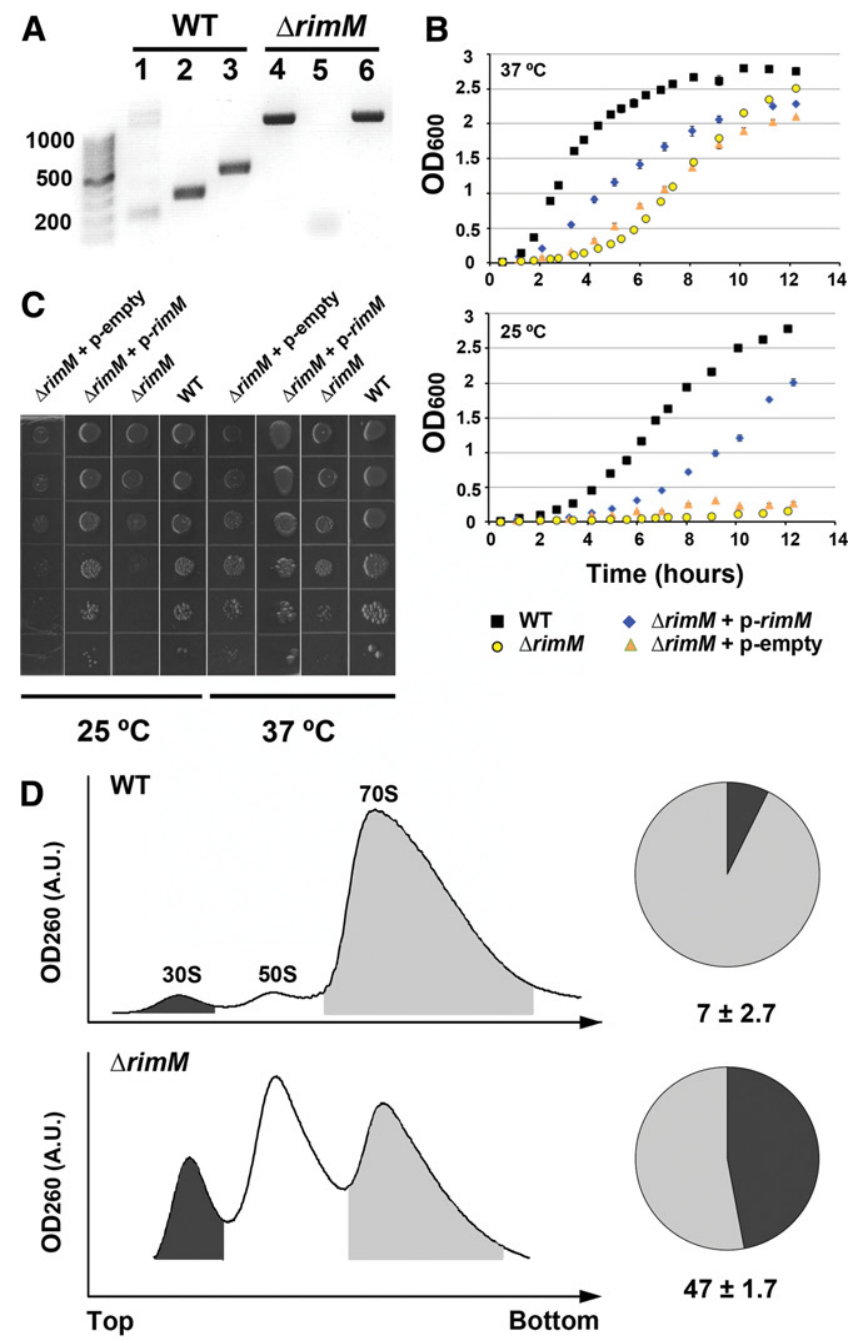

E
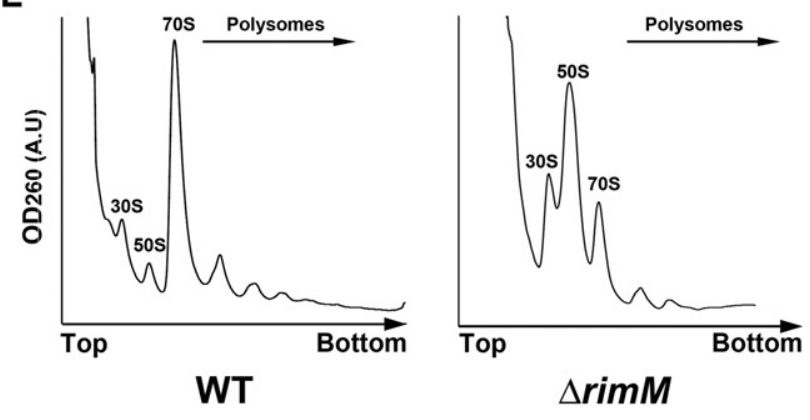

FIGURE 1. (Legend on next page) 
TABLE 1. Doubling time and growth rates of the E. coli BW25113 strains in LB liquid media

\begin{tabular}{|c|c|c|c|c|}
\hline & \multicolumn{2}{|c|}{ Doubling time (DT) (h) } & \multicolumn{2}{|c|}{ Growth rate, $k=\ln 2 / \mathrm{DT}\left(\mathrm{h}^{-1}\right)$} \\
\hline & $37^{\circ} \mathrm{C}$ & $25^{\circ} \mathrm{C}$ & $37^{\circ} \mathrm{C}$ & $25^{\circ} \mathrm{C}$ \\
\hline Parental (WT) & $0.755 \pm 0.03$ & $1.581 \pm 0.03$ & $0.919 \pm 0.04$ & $0.438 \pm 0.01$ \\
\hline$\Delta$ rimM & $1.96 \pm 0.07$ & $3.656 \pm 0.26$ & $0.354 \pm 0.01$ & $0.190 \pm 0.01$ \\
\hline$\Delta \operatorname{rimM}+p-\operatorname{rim} M$ & $0.95 \pm 0.02$ & $1.89 \pm 0.01$ & $0.725 \pm 0.01$ & $0.365 \pm 0.03$ \\
\hline$\Delta$ rimM + p-empty & $2.85 \pm 0.06$ & $3.90 \pm 0.22$ & $0.242 \pm 0.01$ & $0.177 \pm 0.01$ \\
\hline
\end{tabular}

Standard deviations were calculated from three replicas of the experiment. late unprocessed 17S rRNA-containing 30 S ribosomal subunits in a similar manner to the strain lacking the $y j e Q$ gene. Samples from $\Delta y j e Q$ cells and, in small amounts, those from $\triangle$ rim $M$ cells also contained a lower molecular band recognized by the $16 \mathrm{~S}$ internal probe. This band corresponds to a degradation product or an incorrectly processed $16 \mathrm{~S}$ rRNA (Fig. 2C, asterisk; Himeno et al. 2004; Jomaa et al. 2011a).

The protein complement in the free

In this part of the study, we analyzed the rRNA content in the $\Delta$ rim $M$ strain and compared it to the $\Delta y j e Q$ and parental strains from the Keio collection. Purification and visualization of the total RNA from these strains in polyacrylamide gels (Fig. 2A) showed that the parental strain mainly contained fully processed $16 \mathrm{~S}$ and $23 \mathrm{~S}$ rRNAs. The $\Delta y j e Q$ and $\triangle$ rimM cells contained the bands corresponding to the $16 \mathrm{~S}$ and 23S rRNA molecules, but they also accumulated an additional rRNA molecule of a slightly larger size than the $16 \mathrm{~S}$ rRNA. This molecule was present in low abundance $(<5 \%)$ in the sample from the parental strain. In the case of the parental and $\Delta y j e Q$ cells, this molecule has been previously identified as 17S rRNA (Jomaa et al. 2011a), but for the $\Delta$ rimM cells, we performed the identification of this band by Northern blot hybridization. To this end, total rRNA samples from parental, $\Delta y j e Q$ and $\Delta$ rim $M$ cells were resolved in a polyacrylamide gel and transferred to a membrane, which was hybridized with oligonucleotide probes complementary to specific sequences in the $5^{\prime}$ end or the $3^{\prime}$ precursor sequences of $17 \mathrm{~S}$ rRNA. In addition, we also used a probe corresponding to a short internal region of $16 \mathrm{~S}$ rRNA (Fig. 2B). Both precursor sequence probes hybridized with the rRNA band of slightly higher molecular weight than the 16S rRNA, identifying it as $17 \mathrm{~S}$ rRNA. The $16 \mathrm{~S}$ internal probe hybridized to both the 17S rRNA band as well as the lower molecular weight band confirming their identity as $16 \mathrm{~S}$ rRNA (Fig. 2C). These results confirmed that $\triangle$ rimM cells from the Keio collection accumu-
30 subunits that accumulate in the $\Delta$ rim $M$ cells was analyzed by iTRAQ (isobaric tag for relative and absolute quantification) method (Ross et al. 2004). This is the same technique that was previously used to study the protein complement in the free $30 \mathrm{~S}$ subunits that accumulate in $\Delta y j e Q$ cells (Jomaa et al. 2011a), allowing a side-by-side comparison of both $30 \mathrm{~S}$ ribosomal subunits. Here, we performed the iTRAQ double duplex isobaric labeling experiment that compares the relative amount of $\mathrm{r}$-proteins in the free $30 \mathrm{~S}$ subunits from $\triangle$ rimM cells with respect to the mature $30 \mathrm{~S}$ subunits from the parental strain. Free $30 \mathrm{~S}$ subunits from $\triangle$ rimM cells were purified under associating conditions. In the case of the parental strain, mature 30 S subunits were first dissociated from the 50S subunits and then purified (see Materials and Methods section). Results from this analysis are shown in Figure 2D and expressed as $\Delta$ rimM/WT ratios for the r-proteins of the $30 \mathrm{~S}$ subunit. These average ratios were subsequently plotted in the Nomura 30 S subunit assembly map (Fig. 2E) and displayed along with a similar analysis for the $\Delta y j e Q$ 30S subunits (Jomaa et al. 2011a).

Our analysis identified all the r-proteins in the 30S subunit except S1. This protein does not withstand the highsalt washes used during our ribosomal purification (Jomaa et al. 2011a). This result was expected, and thus r-protein S1 was not included in the analysis. We found that most of the primary and secondary $r$-proteins in the free $30 \mathrm{~S}$ subunits purified from $\triangle$ rimM cells were represented at similar levels

FIGURE 1. Cold-sensitive phenotype and ribosome profiling of the $\triangle$ rimM E. coli strain. (A) PCR screening of the $\Delta$ rimM strain. The image shows the obtained PCR products loaded in a 1\% agarose gel. The wild-type parental E. coli BW25113 strain shows a defined 400-bp product amplified by RimM primers that anneal in a sequence internal to the intact rimM gene (lane 2). Using the set of primers annealing sequences upstream and downstream, the rim $M$ gene produced a product of $600 \mathrm{bp}$ corresponding to the complete 549-bp rimM gene plus $50 \mathrm{bp}$ from the flanking sequences (lane 3). In contrast, the $\triangle$ rimM strain has a well-defined band of $\sim 1.2 \mathrm{~kb}$ amplified by primers annealing in sequences $\mathrm{P} 1$ and $\mathrm{P} 2$ flanking the kanamycin resistance cassette introduced during gene deletion (lane 4). This set of primers only produced various unspecific bands of different sizes with the parental strain (lane 1). There was no amplification with RimM primers internal to the rimM gene (lane 5); however, using the set of primers annealing rim $M$ flanking sequences showed a large band of slightly $>1.2 \mathrm{~kb}$ corresponding to the successfully inserted kanamycin resistance cassette flanked by these sequences (lane 6). (B) Growth profiles of the parental and $\Delta$ rim $M$ strain in LB liquid media. The growth profile of the $\Delta$ rimM strain is shown for the untreated strain as well as after being transformed with an empty high-copy plasmid pCA24N (labeled as p-empty) or having reintroduced the rimM gene in this vector (labeled as p-rimM). (C) Dilution plating experiment of saturated cultures of $\Delta$ rim $M$ and parental strains. The $\Delta$ rimM strain complemented with the pCA24N empty vector or encoding the rimM gene are also shown. Cultures were diluted in 10-fold increments, spotted on LB agar plates, and incubated at the indicated temperature. $(D)$ Ribosomes from the parental (top panel) and $\Delta$ rimM strain were fractionated on $10 \%-$ $30 \%$ sucrose gradients, providing the sedimentation profiles shown in the figure. Peaks corresponding to the ribosomal subunits and $70 \mathrm{~S}$ ribosomes are labeled. Peak area for the $30 \mathrm{~S}$ subunit (shaded in black) was measured with respect to the area under the $70 \mathrm{~S}$ peak (shaded in gray) to calculate the percentage of free $30 \mathrm{~S}$ subunit in both strains (pie charts in the right panels). (E) The effects of the rimM deletion are shown in the polysome profiles. Ribosome particles are labeled above the corresponding peaks. 

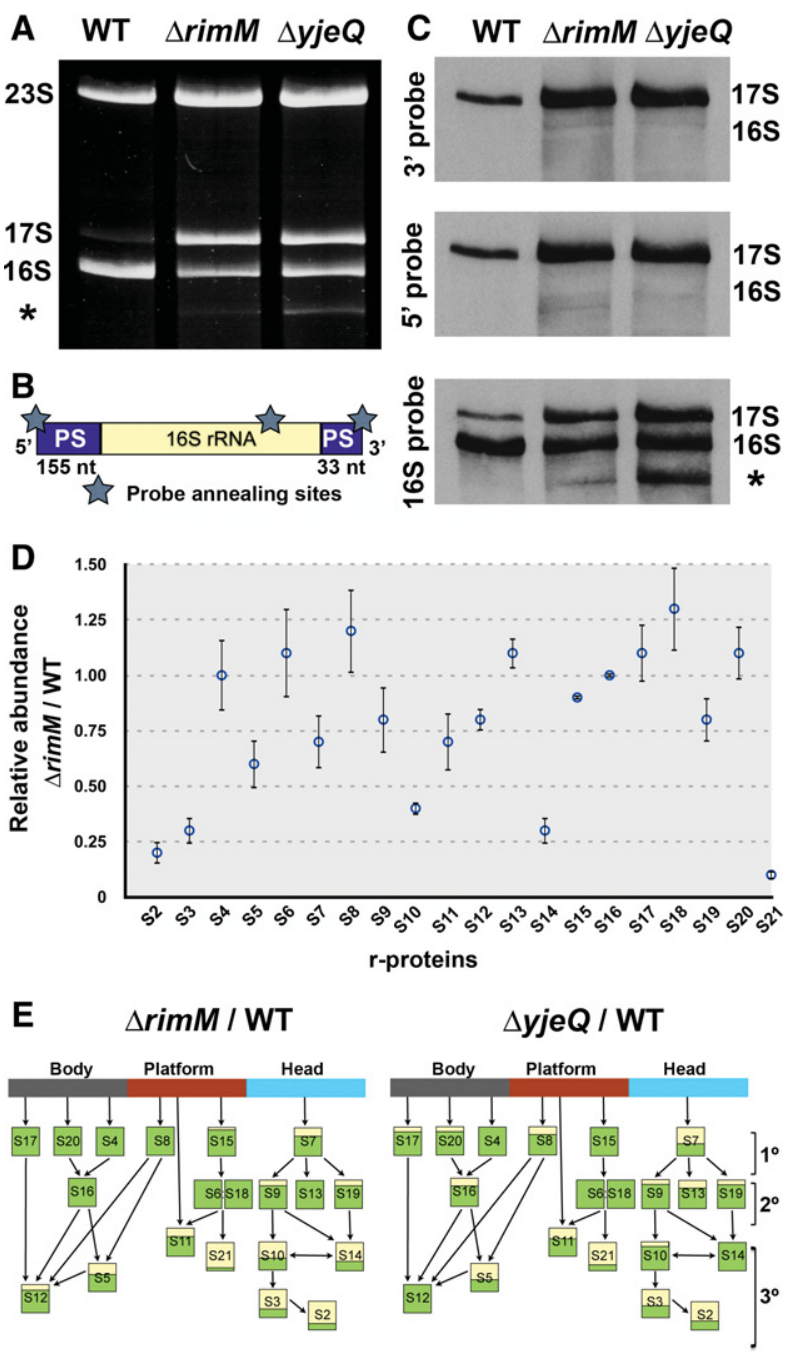

FIGURE 2. Ribosomal RNA and protein complement of immature $30 \mathrm{~S}$ subunits accumulated in the $\triangle$ rimM strain. (A) Total rRNA purified from $\triangle$ rim $M$ and $\triangle y j e Q$ cells resolved in a polyacrylamide gel and stained with ethidium bromide. The 23S, 16S, and 17S rRNA bands are indicated. $(B)$ Diagram of the $17 \mathrm{~S}$ rRNA molecule showing the $5^{\prime}$ and $3^{\prime}$ precursor sequences. The stars indicate the annealing sites for the oligonucleotide probes used in the Northern blot analysis. PS stands for precursor sequence. $(C)$ The identity of small ribosomal rRNA species in the wild-type, $\Delta \operatorname{rim} M$, and $\triangle y j e Q$ strains from the Keio collection was revealed by Northern blot hybridization using $3^{\prime}$ end DIG-dUTP-labeled oligonucleotide probes visualized using a chemiluminescent reaction. The $16 \mathrm{~S}$ and $17 \mathrm{~S}$ rRNA bands are labeled. The asterisk in $A$ and $C$ indicates a lower molecular band corresponding to a degradation product or incorrectly processed $16 \mathrm{~S}$ rRNA (Jomaa et al. 2011a). (D) iTRAQ analysis of the $30 \mathrm{~S}$ subunits purified from the $\triangle$ rim $M$ strain under associating conditions. The relative level for each r-protein with respect to wild-type parental cells is expressed as the ratio $\Delta \operatorname{rim} M / \mathrm{WT}$. The ratio for each protein is calculated as the average of the ratios obtained for all the peptides assigned to each r-protein. Error bars represent the standard deviations of the average for each ratio. (E) The $\Delta$ rimM/WT average ratios were plotted in the Nomura assembly maps (lower panels) and shown along with a similar analysis performed for the free 30 S subunits purified from the $\triangle y j e Q$ strain under identical conditions (data taken from Jomaa et al. 2011a). The proportion of the box colored in yellow is proportional to the degree of underrepresentation of each r-protein in the $30 \mathrm{~S}$ subunits purified from $\triangle \mathrm{rim} M$ and $\triangle y j e Q$ cells. The groups of primary $\left(1^{\circ}\right)$, secondary $\left(2^{\circ}\right)$, and tertiary $\left(3^{\circ}\right)$ proteins are indicated. compared to those of the mature $30 \mathrm{~S}$ subunits from the parental strain, allowing us to conclude that the free $\Delta$ rimM subunits were immature particles at late stages of assembly. However, tertiary r-proteins that bind to the head domain ( $S 2, \mathrm{~S} 3$, S10, and S14) as well as S21 (Fig. 2D,E), a tertiary protein that binds to the platform domain (Supplemental Fig. S1), showed significant underrepresentation. In addition, the primary (S7) and secondary (S9 and S19) r-proteins also binding to the head domain, as well as the tertiary proteins binding to the body (S5 and S12) and platform (S11) domains, were slightly underrepresented (Supplemental Fig. S1).

Considering that in vivo, S7 nucleates the assembly of all other r-proteins in the head (Bunner et al. 2008; Sykes et al. 2010), it is unlikely that the entry of S7 in the assembling $30 \mathrm{~S}$ subunit is affected. A more plausible interpretation is that S7 may be less tightly bound in the immature particle, and it may have dissociated during purification. Interestingly, S13 and S19, which are believed to form the binding site for RimM to the 30S subunit, were not depleted (S13) or only slightly depleted (S19) in these immature particles. This result indicates that entry of these two r-proteins is not dependent on the presence of RimM. However, entrance of all the other $\mathrm{r}$-proteins in the head domain of the $30 \mathrm{~S}$ subunit was affected proportionally to their hierarchy of binding (i.e., primary and secondary were less depleted than tertiary r-proteins). These results are consistent with a role for RimM in mediating the entrance of the tertiary r-proteins in the head domain of the 30 S subunit.

In addition, we found that r-proteins S8, S13, and S18 had a ratio slightly higher than one indicating that these r-proteins are slightly underrepresented in the mature $30 \mathrm{~S}$ subunits. Dissociating conditions (see Materials and Methods) were used to purify the mature $30 \mathrm{~S}$ subunits from parental cells. These conditions expose the ribosomal particles to low magnesium concentrations $(1.1 \mathrm{mM}$ magnesium acetate), which may have caused partial dissociation of some r-proteins. Instead, exposure to low magnesium concentrations was not necessary to purify the immature $30 \mathrm{~S}$ subunits as they are already dissociated from the $50 \mathrm{~S}$ subunits in $\Delta$ rim $M$ cells.

When comparing the iTRAQ analysis of the free $30 \mathrm{~S}$ subunits accumulated in the $\triangle \mathrm{rim} M$ and $\triangle y j e Q$ cells plotted in the Nomura map (Fig. 2E, bottom panel) common patterns emerge. In both cases, tertiary r-proteins S2, S3, and S21 exhibited the higher degree of depletion. In addition, S7, S9, and S19 were also slightly depleted in both immature 30S subunits. The main difference that we found was that, in the $\triangle y j e Q$ 30S subunits, the primary S8, S20, and S17 and secondary S16 r-proteins, which do not bind to the head domain, were slightly depleted. However, they were at normal levels in $\triangle$ rimM $30 \mathrm{~S}$ subunits. It is not possible to discriminate from the published work (Jomaa et al. 2011a) whether these proteins have a defective entrance to the assembling subunit in the absence of the YjeQ protein or if the proteins partially dissociate during purification. However, the main 
conclusion that stems from this comparison is that both $\triangle$ rimM and $\triangle y j e Q$ cells accumulate free 30 S subunits that have not completed their maturation and are severely depleted in the tertiary r-proteins S2, S3, and S21. These immature $30 \mathrm{~S}$ subunits are not identical in terms of the protein complement; nevertheless, they show a comparable pattern of r-protein depletion.

\section{The immature 30S subunit that accumulates in the rimM and yjeQ null strains from the Keio collection exhibit similar structural defects}

Next, we studied the structure of the immature 30 S subunits that accumulated in $\triangle$ rim $M$ cells by cryo-EM. The first step in our structural characterization was to perform a referencefree maximum likelihood-based 2D image classification of the projection images collected of purified $30 \mathrm{~S}$ subunits from $\Delta$ rim $M$ cells (Supplemental Fig. S2). The 2D class averages obtained in this analysis were matched with $2 \mathrm{D}$ projections from the cryo-EM structure of the mature E. coli 30S subunit (EMDB ID 1775) (Jomaa et al. 2011a). This analysis revealed that the densities for the main structural domains of a $30 \mathrm{~S}$ subunit (head, body, and platform) were properly defined and resembled those in the projections from the mature $30 \mathrm{~S}$ subunits. This result suggested that the immature $30 \mathrm{~S}$ subunits that accumulate in $\Delta$ rim $M$ cells were at late stages of assembly. Alternatively, it was possible that the collected data set was contaminated with particle images representing mature 30 S subunits.

Therefore, the first step to obtain the 3D structure of the immature 30S subunit included a supervised classification step (Valle et al. 2002; Gao et al. 2004) aiming to separate particle images representing mature $30 \mathrm{~S}$ subunits that may have copurified with the immature particles (Supplemental Fig. S3). The two references used in this classification were the cryo-EM structure of the mature E. coli 30 S subunit (EMDB ID 1775) (Jomaa et al. 2011a) (reference 1) and the 3D reconstruction obtained from all the particle images in the data set (reference 2) (Supplemental Fig. S3, top panel). This second reference was obtained by a projection matching approach using the X-ray structure of the E. coli $30 \mathrm{~S}$ subunit (PDB ID: 2AVY) (Schuwirth et al. 2005). The fact that the 2D class averages obtained from our data set resembled the projections from the mature 30 S subunit (Supplemental Fig. S2) justified the use of this X-ray structure for our referencebased alignments.

The similarity of the particle images to the two references was assessed by plotting the difference in cross-correlation coefficients for each particle image against equivalent projections from the two references $(\triangle \mathrm{CC}=\mathrm{CC} 2-\mathrm{CC} 1)$. The obtained distribution was bimodal, with two peaks of similar height and area (Supplemental Fig. S3, middle panel). Initially, the distribution of particles was divided into two groups, using $\Delta \mathrm{CC}=0$ as the cutoff value. Particles in each group were used to produce an initial map by the maximum likeli- hood approach (Scheres et al. 2005b,c). These initial maps were subsequently used to refine the $3 \mathrm{D}$ reconstructions for each group of the data by projection matching techniques. Both maps (Supplemental Fig. S3, bottom panel; only map corresponding to particles with $\Delta \mathrm{CC}>1$ is shown) showed the main characteristic domains of the mature $30 \mathrm{~S}$ subunit. However, these structures clearly differed from the mature $30 \mathrm{~S}$ subunit structure in that most of the density representing helix 44 of the rRNA was missing (helix 44 is highlighted in reference 1 in Supplemental Fig. S3). This result suggested that the percentage of particles representing mature $30 \mathrm{~S}$ subunits contaminating the data set was small. To investigate the presence of mature particles even further, we obtained a $3 \mathrm{D}$ reconstruction only from those particles with the highest similarity (lowest $\Delta \mathrm{CC}$ value) to the mature $30 \mathrm{~S}$ subunit structure (reference 1). To this end, the left peak of the distribution was spilt into two parts at the arbitrary $\Delta C C$ value shown in Supplemental Figure S3. The two 3D maps obtained continued to show features not consistent with the mature structure of the 30S subunit (Supplemental Fig. S3, bottom panel). A similar classification approach was shown to be very effective in discriminating mature from immature particles in electron micrographs of immature $30 \mathrm{~S}$ subunits purified from $\triangle y j e Q$ cells (Jomaa et al. 2011a). Therefore, we concluded that, in the case of $30 \mathrm{~S}$ subunits purified from $\triangle$ rim $M$ cells, the amount of particle images representing mature $30 \mathrm{~S}$ subunits contaminating the data set was minimal.

Consequently, we proceeded to perform a maximum likelihood-based classification (Scheres et al. 2005b,c, 2007; Jomaa et al. 2011a) of the entire data set to explore the conformational variability within the population of immature $30 S$ subunits purified from $\triangle$ rimM cells. We found that these 30 S subunits were mainly present in four conformationally different subpopulations (Fig. 3). The percentage of particles for each class was $22 \%, 27 \%, 24 \%$, and $27 \%$, respectively. Performing maximum likelihood-based classifications assuming the existence of more than four subpopulations consistently produced duplication of the 3D structures displayed in Figure 3 (data not shown). Furthermore, we did not obtain a structure that could be assigned to the population of particles containing the degraded rRNA molecules present in small amounts in $\triangle$ rimM cells (Fig. $2 \mathrm{~A}, \mathrm{C}$, asterisk). It is possible that these particles may not exist as a structurally congruent subpopulation and they were eliminated during the classification and 3D reconstruction process. However, these multiple classification experiments allowed us to conclude that the immature $30 \mathrm{~S}$ subunits purified from $\triangle$ rim $M$ cells mainly exist as four conformational subpopulations. Particles in additional conformations may also coexist in the sample, but they are probably not sufficiently abundant to be detected by our classification approaches.

The four cryo-EM maps produced from the maximum likelihood-based classification experiments were refined to a resolution that ranged between $15 \AA$ and $17 \AA$ (Supplemental Fig. S4). These maps represented late assembly intermediates 

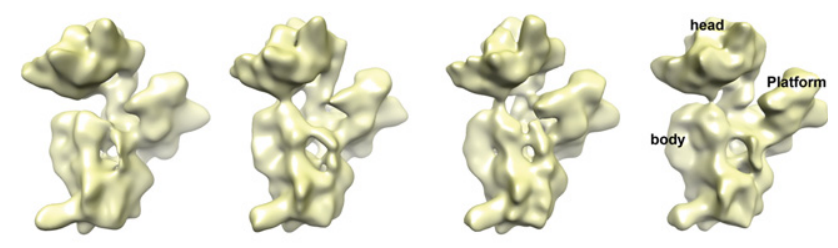

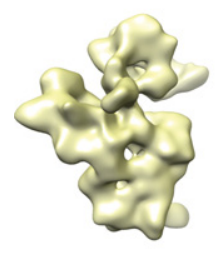

CL 1

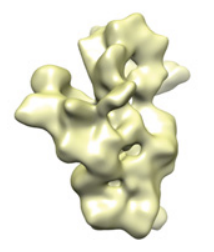

$\mathrm{CL} 2$

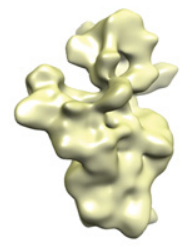

CL 3

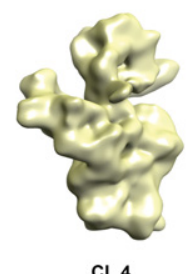

$\mathrm{CL} 4$
FIGURE 3. Conformational subpopulations of immature 30S subunits purified from $\triangle$ rim $M$ cells. Front (top panel) and back (bottom panel) views of the cryo-EM maps representing four conformational subpopulations (labeled CL 1 to CL 4) of immature 30S subunits purified from $\triangle$ rim $M$ cells. Particle classification was obtained using a maximum likelihood-based classification approach. Main domains of the $30 \mathrm{~S}$ subunit are labeled in the structure for subpopulation class 4 . The Fourier shell correlation plots estimating the resolution of these maps are shown in Supplemental Figure S4.

of the 30S subunit, as the main domains of the subunit (head, body, and platform) were present in the structures (Fig. 3).

To assist in the understanding of the conformational differences in the rRNA between these immature particles and the mature $30 \mathrm{~S}$ subunits, the X-ray structure of the mature 30 s subunit (PDB ID 2AVY) (Schuwirth et al. 2005) was docked as a rigid body into the cryo-EM maps obtained for the four conformational subpopulations of the immature 30 S subunits and the mature 30 S subunit (EMDB ID 1775) (Fig. 4A; Jomaa et al. 2011a). The maps corresponding to the immature 30S subunits showed different degrees of distortion in helices 44 and 45 , which are important functional motifs of the rRNA involved in the decoding mechanism (Fig. 4B; Supplemental Fig. S5A). This feature of the cryoEM maps suggested an increased flexibility of these regions. To better estimate the amount of density missing from helices 44 and 45 in each map, we calculated difference maps by subtracting the $3 \mathrm{D}$ maps of the four subpopulations of immature 30S subunits from that of the mature 30S subunit (EMDB ID 1775) (Jomaa et al. 2011a). Most of the densities observed in the difference maps colocalized with r-proteins in the head domain (see below). However, consistent with the distortions observed in helices 44 and 45 , it also showed large densities overlapping with these structural motifs (Fig. 5A; Supplemental Fig. S5B).

The upper domain of helix 44 contributes to the association with the 50S subunit, forming the essential inter-subunit bridge $\mathrm{B} 2 \mathrm{a}$ with the $50 \mathrm{~S}$ subunit. In addition, this region of rRNA constitutes the decoding center, the functional core of the $30 \mathrm{~S}$ subunit. Therefore, these distortions observed in the structures of the immature $30 \mathrm{~S}$ subunit from $\triangle$ rim $M$ cells provide the structural basis to explain previous studies which found these $30 \mathrm{~S}$ subunits were unable to bind the 50S subunit or participate in translation (Bylund et al. 1997). More importantly, these distortions in the decoding center were similar to those previously described in the immature 30 S subunits purified from $\triangle y j e Q$ cells (Jomaa et al. 2011a) from the Keio collection (Fig. 4A, right panel), indicating some degree of structural similarity between the two immature particles.

\section{The immature $\Delta$ rimM $30 \mathrm{~S}$ subunits exhibit structural defects in the head domain consistent with an incomplete protein complement}

In addition to the densities for helix 44 and helix 45 , the difference maps obtained for the four subpopulations of immature 30 S subunits in $\triangle$ rimM cells also showed densities mainly in the head domain that colocalized with several $\mathrm{r}$ proteins (Fig. 5A). In particular, r-proteins S2, S3, and S21 (located in the cleft between the head and the platform), for which ITRAQ analysis determined to be severely underrepresented (Fig. 2D), showed corresponding densities in the difference maps (Fig. 5A). Most of the volume of these r-proteins was enclosed by these densities, consistent with

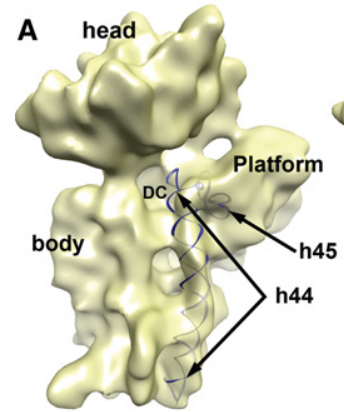

Mature 30S

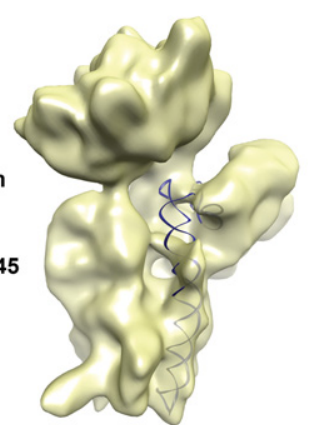

$\Delta y j e Q 30 S$

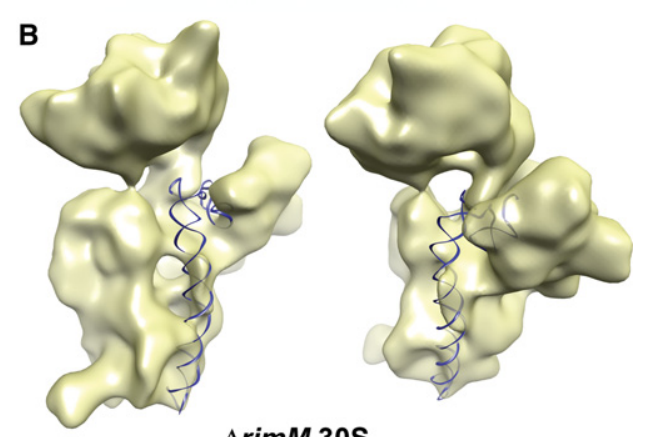

$\Delta \operatorname{rim} M 30 \mathrm{~S}$

FIGURE 4. Structural defects in the $3^{\prime}$ minor domain in the immature $30 \mathrm{~S}$ subunits purified from $\triangle$ rim $M$ cells. (A) Cryo-EM maps of the mature $30 \mathrm{~S}$ subunit and immature $30 \mathrm{~S}$ subunit purified from $\triangle y j e Q$ cells. The X-ray structure of the 16S rRNA (PDB ID 2AVY) was fitted into the cryo-EM maps, and helices 44 and 45 are shown as a ribbon representation. The remaining of the $16 \mathrm{~S}$ rRNA and r-proteins from the Xray structure are not shown for clarity. Main landmarks of the $30 \mathrm{~S}$ subunit are indicated in the left panel. (B) Front and platform view of the cryo-EM map of subpopulation four (CL 4) of the immature 30S subunit purified from $\triangle$ rim $M$ cells. The X-ray structure of the mature $30 \mathrm{~S}$ subunit was docked into the map, and helices 44 and 45 are shown. 
their high degree of underrepresentation in the immature structure (Fig. 5B). However, the densities corresponding to each depleted $r$-protein varied across the difference maps obtained for the four conformational subpopulations of immature 30S subunits (Supplemental Fig. S6). The best correlation was found for the difference map for class 4 . These results demonstrated that our image classification approach was effective in separating immature $30 \mathrm{~S}$ subunits subpopulations exhibiting different degrees of depletion for particular r-proteins.

In the difference maps, we also found densities colocalizing with r-proteins S7, S9, S11, and S19 (Fig. 5A), which iTRAQ analysis found to be slightly underrepresented (Fig. 2D). In these cases, the volume of these proteins enclosed in the corresponding density of the difference map was smaller, consistent with their lower degree of depletion (Fig. 5B). These volumes also varied between the four difference maps obtained from the subpopulations of immature 30S subunits (Supplemental Fig. S7).

In addition, we also found a few exceptions in the correlation between the iTRAQ data and difference map analysis. One of them was the r-protein S13 which, along with S19, is believed to form the binding site for RimM to the $30 \mathrm{~S} \mathrm{sub-}$ unit. The $\mathrm{C}$ terminus of $\mathrm{S} 13$ was enclosed in a corresponding density present in the difference maps (Supplemental Fig. S7). This density was larger in the difference map for class 4 , and in this case, almost the entire volume of r-protein S13 was enclosed in the density (Fig. 5B). However, S13 was not found to be underrepresented according to iTRAQ analysis (Fig. 2D). This result suggested that $S 13$ has a higher degree of flexibility in the immature $30 \mathrm{~S}$ subunits, causing a partial smearing of the corresponding density in the cryo-EM maps of the immature subunits. Alternatively, it is plausible that $S 13$ is more loosely bound in the immature $30 \mathrm{~S}$ subunit and partially dissociates during the dilution or vitrification process that the sample undergoes during cryo-EM. Conversely, we did not find a density for S10 and only a small one for S14 in the difference maps (Fig. 5B; Supplemental Fig. S6). However, these $\mathrm{r}$-proteins were found to be severely underrepresented according to our iTRAQ analysis (Fig. 2D). Similarly, r-proteins S5 and S12, in the body of the 30S subunit, were found to be slightly depleted, but they did not show a corresponding density in the difference maps (Fig. 5A). It is possible that concomitant movements of the r-RNA obscured the absence of these proteins, generating densities in the structures for the immature $30 \mathrm{~S}$ subunits similar to those produced by these r-proteins in the mature subunit.

\section{DISCUSSION}

The defects observed in immature $30 \mathrm{~S}$ subunits purified from $\triangle$ rim $M$ cells suggest a role for RimM in assisting the entrance of tertiary r-proteins that bind to the head domain of the $30 \mathrm{~S}$ subunit, as well as in the folding of the $3^{\prime}$ minor domain of the $16 \mathrm{~S}$ rRNA. More importantly, the distortions observed in the decoding center and r-protein depletion pattern resemble those found in the immature $30 \mathrm{~S}$ subunits that accumulate in $\Delta y j e Q$ cells. These findings suggest that RimM assists in the late stages of assembly of the $30 \mathrm{~S}$ subunit. These results are also consistent with previous data (Bylund et al. 1998, 2001; Inoue et al. 2003, 2006; Campbell and Brown 2008), suggesting that RimM performs its function in conjunction with other assembly factors, including YjeQ.

Early in vitro and in vivo experiments were able to identify discrete assembly intermediates of the $30 \mathrm{~S}$ and $50 \mathrm{~S}$ subunits (Hayes and Hayes 1971; Lindahl 1975; Sieber and Nierhaus 1978; Nierhaus 1980). These early studies seeded the hypothesis that assembly proceeds through a single pathway
A
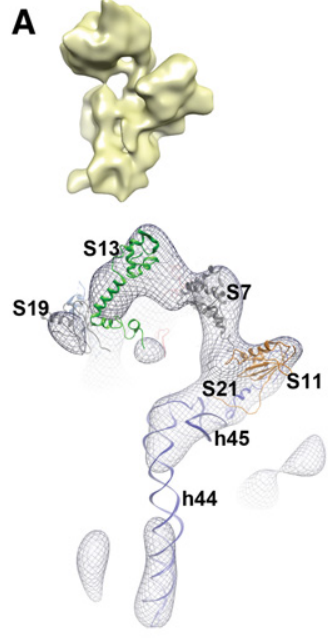

B

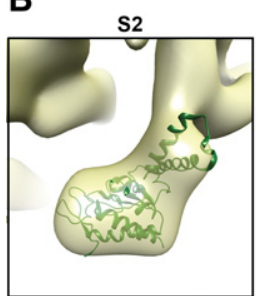

s9

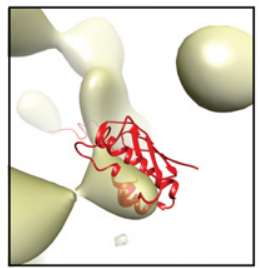

S14

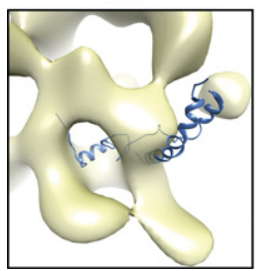

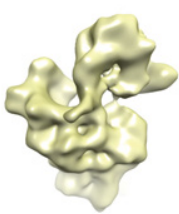

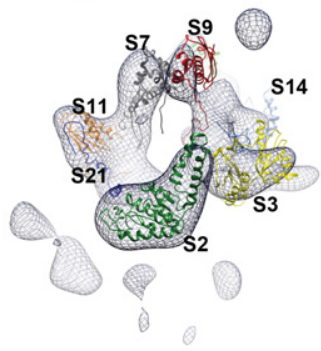

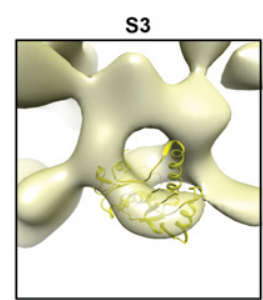

S11

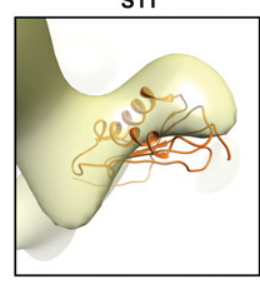

S19

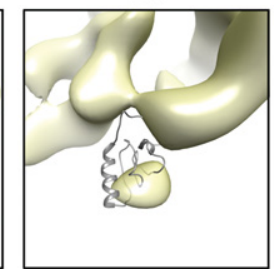

FIGURE 5. (Legend on next page)

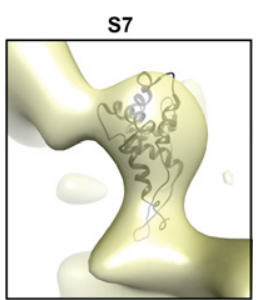

$\mathrm{S} 13$

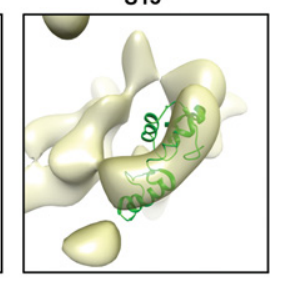

S21

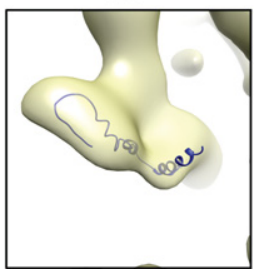


characterized by the existence of rate limiting intermediates. Under this model, it was thought that assembly factors were required to assist the immature particles in overcoming energy barriers along the assembly pathway.

More recently, experiments from the Woodson (Adilakshmi et al. 2008) and Williamson (Talkington et al. 2005; Williamson 2005) laboratories have established that, in the case of the $30 \mathrm{~S}$ subunit, the particle nucleates in multiple points along the rRNA, and assembly proceeds through multiple parallel pathways with no single rate-limiting steps. Under this model, the role of trans-acting factors is to guide the folding landscape of the rRNA and to facilitate productive conformations and specific protein-RNA interactions (Shajani et al. 2011).

A central question to this study is how assembly factors that work in conjunction perform their role and assist the ribosomal assembly if the process follows multiple parallel pathways that occur simultaneously. Herein, we have addressed this question specifically for RimM, which is functionally related to Era, RbfA, and YjeQ.

It is difficult to envision how assembly factors that are known to work in conjunction would assist an assembly process that follows parallel pathways, converging only in the mature state, unless all factors assist every pathway. Alternatively, if each factor is assisting one of the parallel pathways, then elimination of any of these factors would cause accumulation of a different assembly intermediate. In addition, it is most likely that factors acting in one pathway would not bind immature intermediates that accumulate as a result of eliminating a factor involved in a different pathway. This scenario does not agree with the findings presented here or the existing literature (Goto et al. 2011) as described below.

The similarities found between the immature particles that accumulate in the $\triangle$ rim $M$ and $\triangle y j e Q$ strain (Jomaa et al. 2011a) are consistent with a model that we named here as the "early convergency model" (Fig. 6, top panel), in which several parallel assembly pathways converge into a common immature assembly intermediate as opposed to the mature state. Functionally related factors, such as RimM, Era, RbfA, and YjeQ, will bind to this intermediate with a defined hierarchy to catalyze the last steps of maturation. After binding, their mechanism of action may follow a "simultaneous" pattern (i.e., all of them bind to the same intermediate to catalyze together the last maturation steps), a "sequential" pattern (i.e., each factor performs its function independently and is released before the next factor binds to the maturing 30 S subunit) (Fig. 6, bottom panel), or by a combination of these two possibilities (not displayed in Fig. 6).

For RimM, Era, RbfA, and YjeQ, the hierarchy of binding to the immature intermediate or whether their binding follows a simultaneous or sequential model is still unknown. YjeQ (Goto et al. 2011) and RimM (data not shown) are not capable of binding the immature 30 S subunit that accumulates in their respective $y j e Q$ and rimM null strains. However, RbfA binds the immature 30S subunit purified from the $\Delta y j e Q$ strain (Goto et al. 2011). Therefore, it is plausible that RbfA may be the first factor binding the assembling subunit. This initial binding event may allow for the binding of YjeQ, RimM, and Era. Structurally, the immature 30S subunits that accumulate in $\triangle$ rim $M$ cells differ more from the mature 30S subunit structure than the immature particles purified from a $\triangle y j e Q$ strain (Jomaa et al. 2011a). Whether that implies that the particles from $\triangle$ rim $M$ cells constitute an earlier assembly intermediate or that RimM binding to the assembling 30S subunit occurs before YjeQ does still has to be determined. Further experiments beyond the scope of this study will define the hierarchy of binding for these functionally linked factors.

We are mindful that, in addition to RimM, Era, RbfA, and $\mathrm{YjeQ}$, other trans-acting factors assisting the late stages of assembly of the 30 S subunit in bacteria have been described (Wilson and Nierhaus 2007). Undoubtedly, future work will expand the current list of assembly factors and define a network of functional interactions among known and newly established factors. In this context, it is possible that the entire folding landscape of the $30 \mathrm{~S}$ particle may converge to a few common intermediates instead of the mature state. Each group of functionally related factors will target one of these intermediate particles to catalyze the latest steps of maturation.

What are the functional advantages of the early convergency model of assembly for the cell? Tight regulation of the last steps in the maturation process of the ribosome is essential for cell survival under conditions with scarce nutrients (Britton 2009). Translating ribosomes become stalled when amino

FIGURE 5. Structural defects in the head domain in the immature 30 S subunits purified from $\triangle$ rim $M$ cells. (A) Front and back views of the difference map obtained by subtracting the cryo-EM map of the immature 30 S subunits (CL 4) from $\triangle$ rimM cells from that of the mature 30 S subunit (EMDB ID 1775). Densities in the different maps are represented as a mesh, and the X-ray structure of the mature 30S subunit (PDB ID 2AVY) is shown docked into the difference map. The cryo-EM maps above each view are an aid for orientation, and they represent the cryo-EM map of the immature $30 \mathrm{~S}$ subunit in the same view as the difference map below. The difference map showed densities overlapping with helices 44 and 45 of the 16 rRNA from the docked X-ray structure of the $30 \mathrm{~S}$ subunit, indicating that these structural motifs do not have a correspondent density in the cryo-EM maps of the immature $30 \mathrm{~S}$ subunits. The densities in the head domain of the difference map colocalized with the r-proteins from the structure of the mature $30 \mathrm{~S}$ subunit that iTRAQ analysis identified as underrepresented in the immature $30 \mathrm{~S}$ subunits from $\triangle$ rimM cells. (B) Images in this panel provide a detailed view of the docking of underrepresented r-proteins from the X-ray structure of the mature $30 \mathrm{~S}$ subunit into the densities of the difference map. A higher proportion of a r-protein being enclosed in the density of the difference map indicates a larger amount of the corresponding density for that r-protein missing in the cryo-EM map of the immature $30 \mathrm{~S}$ subunit. The densities in the difference map are shown as a semitransparent surface. The cryo-EM map for subpopulation 4 of immature $30 \mathrm{~S}$ subunits was used to produce the difference map displayed in this panel. A similar difference map analysis performed with the cryo-EM maps for the other three subpopulations is shown in Supplemental Figures S5, S6, and S7. 


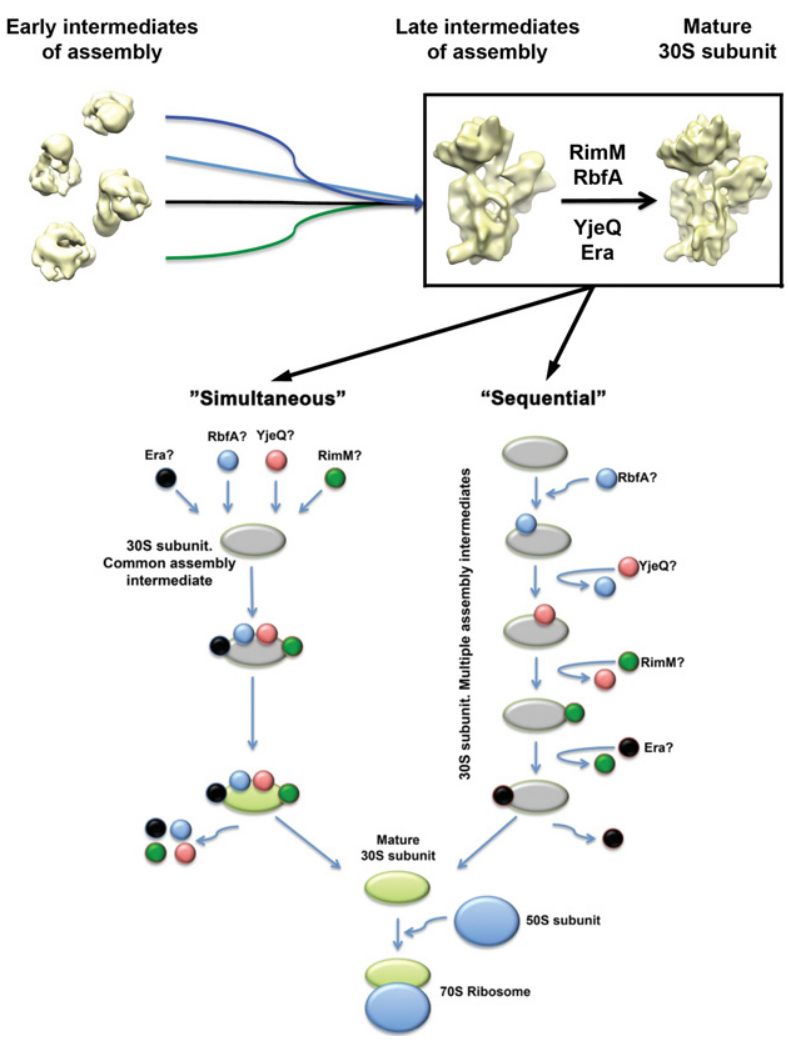

FIGURE 6. Early convergence model for the assembly of the $30 \mathrm{~S}$ subunit. The "early convergency model" presented in this diagram (top panel) suggests that multiple parallel assembly pathways converge into a late assembly intermediate. A group of functionally related assembly factors (RimM, RbfA, YjeQ, and Era) will target this intermediate and catalyze the latest steps of maturation. Binding of these factors to the assembly intermediate will occur following a defined hierarchy. The bottom panel illustrates the two alternative models for the mechanism of action of RimM, RbfA, YjeQ, and Era. In the "simultaneous" model, all these assembly factors bind to the same intermediate to catalyze together the last maturation steps. In an alternative model, each factor performs its function independently and is released before the next factor binds to the maturing 30 S subunit. A "hybrid" model is also possible (not displayed) where some factors may function sequentially, and others may act simultaneously. The question mark beside the name of each factor indicates that the order of binding is unknown.

acids are depleted, but removal and degradation of partially translated proteins represents an important energy cost for the cell during times of limited resources. Therefore, the cell needs to have regulatory mechanisms in place to minimize the number of ribosomes that engage in translation under such conditions. Convergence of multiple parallel assembly pathways into a late assembly intermediate may provide the cell with an efficient mechanism to arrest assembly just before the ribosomal subunit reaches the mature state. Under the early convergency model, the final maturation steps will be catalyzed by functionally related factors (RimM, Era, RbfA, and $\mathrm{YjeQ}$ ) limiting the number of $30 \mathrm{~S}$ subunits that have the ability to engage in protein translation. Most importantly, it may create a reservoir of immature ribosomal subunits on "standby" that can complete their maturation process and become readily available for protein translation as soon as nutrients become available. Considering that some of these factors are GTPases (YjeQ and Era) (Daigle et al. 2002; Sharma et al. 2005), this mechanism could allow the cell to couple ribosomal assembly with intracellular GTP concentrations (Potrykus and Cashel 2008). A decrease in the GTP/GDP ratio would increase the amount of the GDP-bound form of the GTPase factors, inhibiting association with the 30 S subunit and preventing catalysis of the last step of maturation of the ribosomal particle. Conversely, if assembly occurs through multiple parallel pathways converging only in the mature state, the cell needs to shut down each single pathway independently to completely stop production of mature ribosomal subunits. It is arguable whether a tight control on the amount of mature subunits produced by the cell is possible under the late convergency model.

\section{MATERIALS AND METHODS}

\section{PCR screening of the $\Delta r i m M$ Keio collection strain}

The primers used for the analysis were as follows:

P1 5'-ATTCCGGGGATCCGTCGACC-3'

P2 5'-TGTAGGCTGGAGCTGCTTCG-3'

RimM Rev 5'-TTCGTGGGTGGCTCAGAGTGTTTTC-3'

RimM Fwd 5'-CGGTACGAGACGTTCCTTGATACCA-3'

RimM flanking $15^{\prime}$-GCCGTCTTTTACCGTTTATCCGGTG-3'

RimM flanking 2 5'-CAAAGCAGCTTAATCTGTCACGGTG-3'

In the Keio collection, the kanamycin resistance cassette introduced during gene deletion is flanked by 20-nt-long upstream (P1) and downstream (P2) sequences (Baba et al. 2006) that are the same as in our $\mathrm{P} 1$ and $\mathrm{P} 2$ primers. These primers anneal to the $\mathrm{P} 1$ and P2 sequences and amplify the entire kanamycin resistance cassette when present $(\sim 1200 \mathrm{bp})$. The RimM Rev and RimM Fwd set of primers was designed to anneal within the sequence of the rim $M$ gene and produce a product of $400 \mathrm{bp}$. Finally, the RimM flanking 1 and 2 primers correspond to upstream and downstream sequences from the rimM gene, respectively, and they were used to positively identify a product the size of the gene (549 bp +50 $\mathrm{bp})$ or the cassette inserted into the same region $(\sim 1.2 \mathrm{~kb}+50 \mathrm{bp})$.

Template DNA samples were prepared from the wild-type parental E. coli BW25113 and $\Delta$ rimM strains. Strains were grown overnight in 3-mL cultures of Luria-Bertani (LB) media (with $30 \mu \mathrm{g} /$ $\mathrm{mL}$ of kanamycin for $\triangle$ rimM). Cell pellets were resuspended in 50 $\mu \mathrm{L}$ of TNE buffer (50 mM Tris- $\mathrm{HCl}, \mathrm{pH} 8,100 \mathrm{mM} \mathrm{NaCl}$, and $0.1 \mathrm{mM}$ EDTA). Lysis was performed by adding $5 \mu \mathrm{L}$ of a $10 \mathrm{mg} /$ $\mathrm{mL}$ solution of lysozyme and incubating the cells on ice first for $30 \mathrm{~min}$ and then heating at $65^{\circ} \mathrm{C}$ for $10 \mathrm{~min}$. Genomic DNA was extracted by adding $50 \mu \mathrm{L}$ of equilibrated phenol-chloroform $(1: 1 \mathrm{v} / \mathrm{v})$, and the mixture was centrifuged for $3 \mathrm{~min}$ at $10,000 \mathrm{~g}$ at room temperature. The upper phase was recovered and the DNA precipitated by adding an equal volume of ice-cold $100 \%$ ethanol. DNA was pelleted by centrifugation at $10,000 \mathrm{~g}$ for $20 \mathrm{~min}$ at $4^{\circ} \mathrm{C}$. DNA pellets were washed with ice-cold $70 \%$ ethanol and subsequently centrifuged at $10,000 \mathrm{~g}$ at $4^{\circ} \mathrm{C}$ for $5 \mathrm{~min}$. DNA pellets were dried and then resuspended in $100 \mu \mathrm{L}$ sterile water for use in PCR. 
The PCR was performed with the following cycle times and temperatures: 5 min DNA template melting at $95^{\circ} \mathrm{C}$ and 30 cycles of amplification ( $1 \mathrm{~min}$ DNA template melting at $95^{\circ} \mathrm{C}, 1 \mathrm{~min}$ annealing at $63^{\circ} \mathrm{C}, 2 \mathrm{~min}$ elongation at $72^{\circ} \mathrm{C}$ ), followed by a final 20 -min elongation step at $72^{\circ} \mathrm{C}$. The completed PCR reactions were resolved on a $1 \%$ agarose gel in TE buffer (50 mM Tris- $\mathrm{HCl}, \mathrm{pH} 8.0$ and $0.1 \mathrm{mM}$ EDTA), stained with GelRed (Biotium) and visualized with a UV transilluminator.

\section{Culture growth conditions}

To obtain the growth curves, the $\Delta$ rim $M$ and parental strains were inoculated in $50 \mathrm{~mL}$ of LB media from overnight cultures at a 1:100 dilution. Cells were incubated at either $25^{\circ} \mathrm{C}$ or $37^{\circ} \mathrm{C}$ with shacking, and culture density was monitored by measuring the optical density at $600 \mathrm{~nm}\left(\mathrm{OD}_{600}\right)$. To reintroduce the RimM protein into $\Delta$ rimM cells, we used the high-copy plasmid pCA24N. This vector expresses $\mathrm{N}$-terminal histidine-tagged RimM under the control of isopropylbeta-D-thiogalactopyranoside-inducible promotor $\mathrm{P}_{\mathrm{T} 5 \text {-lac }}$. This expression plasmid was purified from the corresponding clone from the ASKA library collection, which contains a complete set of open reading frame clones of E. coli (Kitagawa et al. 2005). The purified pCA24N plasmid (empty or expressing RimM) was transformed into the $\Delta$ rimM cells using standard protocols (Sambrook et al. 1989) before proceeding to obtain the growth curves as described above. Doubling times of cultures were calculated as DT $=(\mathrm{t} 2-\mathrm{t} 1)$ $\times\left[\log 2 /\left(\log \mathrm{OD}_{600} @ \mathrm{t} 2 / \log \mathrm{OD}_{600} @ \mathrm{t} 1\right)\right]$ and expressed in hours. Growth rates were expressed as $k=\ln 2 / \mathrm{DT}$ and expressed in $\mathrm{h}^{-1}$.

Dilution plating experiments were performed by inoculating 0.5 $\mathrm{mL}$ of a saturated overnight culture in $50 \mathrm{~mL}$ of fresh $\mathrm{LB}$ media. Cultures were grown at $37^{\circ} \mathrm{C}$ with shaking until an $\mathrm{OD}_{600}$ of 0.2 . Then, serial dilutions were made in 10-fold increments, and $5 \mu \mathrm{L}$ of each dilution was immediately spotted onto LB agar plates and incubated at either $37^{\circ} \mathrm{C}$ or $25^{\circ} \mathrm{C}$. The plates were incubated for $\sim 18$ and 40 h, respectively, or until isolated colonies of the parental strain reached $\sim 2 \mathrm{~mm}$ in diameter.

\section{S subunit purification and sucrose gradient ultracentrifugation analysis}

For each strain, $1 \mathrm{~L}$ of $\mathrm{LB}$ was inoculated with $10 \mathrm{~mL}$ of saturated overnight culture and grown to an $\mathrm{OD}_{600}$ of 0.2 . Cultures were then cooled to $4^{\circ} \mathrm{C}$, and subsequent steps were carried out at this temperature. Bacteria were harvested at $5000 \mathrm{~g}$ for $10 \mathrm{~min}$ and the pellets resuspended in buffer A (20 mM Tris- $\mathrm{HCl}, \mathrm{pH} 7.5,10 \mathrm{mM}$ magnesium acetate, $100 \mathrm{mM} \mathrm{NH} 4 \mathrm{Cl}, 0.5 \mathrm{mM}$ EDTA, and $3 \mathrm{mM}$ 2-mercaptoethanol) with the addition of Roche Complete Mini protease inhibitor cocktail and DNaseI (Roche). Resuspended cells were then lysed by pressurized extruder or by four passes in a French press at 20,000 lbs/in ${ }^{2}$. The cell lysate was centrifuged at $30,000 \mathrm{~g}$ for 40 min to clear cell debris. Clarified lysate (S30 fraction) was layered over a 1.1 M sucrose cushion of equal volume, composed of buffer B (20 mM Tris- $\mathrm{HCl}$ at $\mathrm{pH} 7.5,10.5 \mathrm{mM}$ magnesium acetate, 500 $\mathrm{mM} \mathrm{NH} 4 \mathrm{Cl}$, $0.5 \mathrm{mM}$ EDTA, $3 \mathrm{mM}$ 2-mercaptoethanol) and centrifuged for $16 \mathrm{~h}$ at $100,000 \mathrm{~g}$. The ribosome pellet was resuspended in buffer $\mathrm{C}(10 \mathrm{mM}$ Tris- $\mathrm{HCl}$ at $\mathrm{pH} 7.5,10.5 \mathrm{mM}$ magnesium acetate, $100 \mathrm{mM} \mathrm{NH} 4 \mathrm{Cl}, 0.5 \mathrm{mM}$ EDTA, and $7 \mathrm{mM}$ 2-mercaptoethanol) and pelleted again at $100,000 \mathrm{~g}$ for $16 \mathrm{~h}$.
The washed ribosome pellet was resuspended in buffer $\mathrm{E}(10 \mathrm{mM}$ Tris-HCL, pH 7.5, $10 \mathrm{mM} \mathrm{Mg}$ acetate, $60 \mathrm{mM} \mathrm{NH}_{4} \mathrm{Cl}$, and $3 \mathrm{mM} \mathrm{2-}$ mercaptoethanol). Ribosomal subunits remain associated under these buffer conditions. Sixty to $80 \mathrm{~A}_{260}$ units of resuspended crude ribosomes were then applied to $34 \mathrm{~mL} 10 \%-30 \%$ (w/v) sucrose gradients prepared with Buffer $\mathrm{E}$. The gradients were centrifuged for 16 $\mathrm{h}$ at $40,000 \mathrm{~g}$ on a Beckman SW32 Ti rotor. Gradients were fractionated using a Brandel fractionator apparatus and an AKTAprime FPLC system (GE Healthcare). The elution profile was monitored by $\mathrm{UV}$ absorbance at $\mathrm{A}_{260}$, and fractions corresponding to the $30 \mathrm{~S}$ subunit peak were pooled and collected for cryo-electron microscopy and ITRAQ analysis. The $30 \mathrm{~S}$ subunits were removed from the sucrose buffer by centrifuge spinning at $100,000 \mathrm{~g}$ for $16 \mathrm{~h}$, after which the pellet was rinsed, resuspended, and stored at $-80^{\circ} \mathrm{C}$ in buffer E. Sample concentration was determined by sample absorbance at $260 \mathrm{~nm}$, where $1 \mathrm{~A}_{260}$ unit is equivalent to $69 \mathrm{pmol}$ of $30 \mathrm{~S}$.

The purified $30 \mathrm{~S}$ subunits from the parental E. coli strain were purified using the same protocol. However, the ribosome pellet was resuspended in buffer $\mathrm{F}$ ( $10 \mathrm{mM}$ Tris- $\mathrm{HCl}$, pH 7.5, $1.1 \mathrm{mM}$ magnesium acetate, $60 \mathrm{mM} \mathrm{NH} 4 \mathrm{Cl}, 0.5 \mathrm{mM}$ EDTA, and $2 \mathrm{mM}$ 2-mercaptoethanol), and the sucrose gradients were also prepared in this buffer. The final purified $30 \mathrm{~S}$ subunits were resuspended in buffer $\mathrm{E}$.

The proportion of free $30 \mathrm{~S}$ to bound $30 \mathrm{~S}$ (i.e., $30 \mathrm{~S}$ subunits in $70 \mathrm{~S}$ binary complexes) in the $\Delta$ rim $M$ and parental strains was calculated using the $30 \mathrm{~S}$ and $70 \mathrm{~S}$ peak areas of the sucrose gradient profiles. The area of the $30 \mathrm{~S}$ peak plus one-third the area of the $70 \mathrm{~S}$ peak corresponds to the total $30 \mathrm{~S}$ population as it contributes to the absorbance. The area of the $30 \mathrm{~S}$ peak was divided by the total $30 \mathrm{~S}$ absorbance to obtain the percentage of free $30 \mathrm{~S}$.

To obtain the polysome profiles (Ron et al. 1966), 100-mL cultures of the strains were grown in $\mathrm{LB}$ at $37^{\circ} \mathrm{C}$ to an $\mathrm{OD}_{600}$ of 0.2 . Polysomes were stabilized by addition of chloramphenicol (Brow and Noller 1983 ) to a final concentration of $0.1 \mathrm{mg} / \mathrm{mL}$ and continued to shake for 2-3 min before the cultures were harvested in two conical tubes by centrifugation at $5000 \mathrm{~g}$ for $10 \mathrm{~min}$. Each pellet was resuspended in $0.5 \mathrm{~mL}$ of cold buffer containing $20 \mathrm{mM}$ Tris-HCl, pH 7.5, $15 \mathrm{mM}$ $\mathrm{MgCl}_{2}$, and transferred to plastic centrifuge tubes. Lysozyme was added to a final concentration of $1 \mathrm{mg} / \mathrm{mL}$ and the tubes incubated on ice for $15 \mathrm{~min}$. The suspension was frozen in liquid nitrogen and then thawed slowly in a water bath at $30^{\circ} \mathrm{C}$ until melted. After three cycles of freezing and thawing, cell lysis was completed by adding $15 \mu \mathrm{L}$ of $10 \%$ sodium deoxycholate and $10 \mu \mathrm{L}$ of DNase I $(1 \mathrm{mg} /$ $\mathrm{mL}$ ). The mixture was spun at $5000 \mathrm{~g}$ for $15 \mathrm{~min}$, and the supernatant was transferred to new plastic tubes. The sample concentration was determined by absorbance at $260 \mathrm{~nm}$, and $10 \mathrm{~A}_{260}$ units of resuspended crude ribosomes were then applied to $10 \mathrm{~mL} 10 \%-40 \%$ $(\mathrm{w} / \mathrm{v})$ sucrose gradients prepared in buffer containing $10 \mathrm{mM}$ Tris$\mathrm{HCl}, \mathrm{pH} 7.5,50 \mathrm{mM} \mathrm{KCl}, 10 \mathrm{mM} \mathrm{MgCl}_{2}$, and $6 \mathrm{mM}$ 2-mercaptoethanol. The gradients were spun for $3.5 \mathrm{~h}$ at $21,000 \mathrm{~g}$ on a Beckman SW41 Ti rotor at $4^{\circ} \mathrm{C}$. Gradients were fractionated using a Brandel fractionator apparatus and a syringe pump. The elution profile was monitored by UV absorbance at $\mathrm{A}_{260}$.

\section{Analysis of cellular rRNA}

Overnight cultures of the parental and $\triangle$ rim $M$ strains were inoculated at a 1:100 dilution into fresh $\mathrm{LB}$ media and grown at $37^{\circ} \mathrm{C}$ with shaking to an $\mathrm{OD}_{600}$ of 0.2 . Samples of $2 \mathrm{~mL}$ were then taken from the cultures and pelleted in a microcentrifuge at $9000 \mathrm{~g}$ for $10 \mathrm{~min}$. 
The rRNA from each sample was purified using the RNeasy mini kit (Qiagen) according to the manufacturer's protocol. RNA concentration in the sample was then measured by $\mathrm{A}_{260}$, where 1 absorbance unit is equivalent to $40 \mu \mathrm{g} / \mathrm{mL}$ of RNA. Approximately $2 \mu \mathrm{g}$ of purified RNA samples were loaded on an $8 \mathrm{M}$ urea, $4 \%$ [w/v] polyacrylamide gel in $1 \times$ Tris-borate-EDTA (TBE) assembled in an adjustable slab gel apparatus (CBS Scientific Co.). Samples were resolved by electrophoresis at $300 \mathrm{~V}$ for $16 \mathrm{~h}$ and then stained with ethidium bromide in $0.5 \times$ TBE for visualizing on a UV transilluminator.

Northern hybridization analysis followed the manufacturer's protocol for the DIG (Digoxigenin) chemiluminescent nucleotide detection (Roche). The rRNA resolved on the $8 \mathrm{M}$ urea, $4 \%[\mathrm{w} / \mathrm{v}]$ polyacrylamide gel was transferred to a nitrocellulose membrane using a Bio-Rad Transblot semi-dry transfer system at 25V for $45 \mathrm{~min}$ and fixed by exposing the membrane to UV light $(302 \mathrm{~nm})$ for 1.5 min. The membranes were equilibrated in Roche Easy-Hyb solution at $50^{\circ} \mathrm{C}$ for $30 \mathrm{~min}$ and then hybridized with $0.1-1 \mathrm{pmol} / \mathrm{mL} 3^{\prime}$ end DIG-dUTP-labeled oligonucleotide probes in Easy-Hyb solution containing $0.1 \mathrm{mg} / \mathrm{mL}$ poly-adenosine at $50^{\circ} \mathrm{C}$, overnight. The sequences for the probes were as follows:

\section{$5^{\prime}$ probe (5'-TTAAGAATCCGTATCTTCGAGTGCCCACA-3')} $3^{\prime}$ probe (5'-TGTGTGAGCACTGCAAAGTACGCTTCTTTAA GGTAAGG-3') and 16S internal probe (5'-GGGCCATGATGA CTTGACGTCATCCCCACC-3')

Labeling of the oligonucleotides at the $3^{\prime}$ end was performed with DIG Oligonucleotide Tailing Kit 2nd generation (Roche) following manufacturer protocols.

The hybridized membrane was washed, blocked, and developed for chemiluminescence according to manufacturer protocols (Roche). Membranes were exposed to Kodak BioMax XAR film, which was subsequently developed.

Membranes were first typically hybridized and developed with the $3^{\prime}$ probe, followed by subsequent hybridizations with the $5^{\prime}$ and $16 \mathrm{~S}$ probes. A membrane stripping procedure was performed on the membranes between hybridizations. The probe was stripped by rinsing the membrane first with distilled water and then with boiling $0.1 \%$ SDS solution for $10 \mathrm{~min}$. The membrane was then rinsed with wash buffer (100 mM maleic acid, $150 \mathrm{mM} \mathrm{NaCl}, \mathrm{pH} 7.5,0.3 \%$ [v/v] Tween 20) before hybridization with the next probe.

\section{iTRAQ analysis}

The 30 S subunits from parental and $\triangle$ rimM E.coli cells were purified under dissociating and associating conditions, respectively. Total protein concentration in the samples was measured using the BioRad Protein Assay (Bio-Rad). A total of $15 \mu \mathrm{g}$ of protein was taken from each 30 S subunit purification, precipitated with $80 \%$ ice-cold acetone, and washed twice with $100 \%$ acetone. The pellet was resuspended in buffer containing $0.5 \mathrm{M}$ triethylammonium bicarbonate, $0.01 \%$ SDS, followed by protein reduction with Tris (2-carboxyethyl) phosphine and cysteine blockage with methylmethanethiosulfate. Samples were then digested with trypsin as described previously (Jiang et al. 2006).

Labeling of the peptides with the iTAQ reagents was done according to manufacturer protocols (AB SCIEX). The iTRAQ reagents 117 and 114 were used to label tryptic peptides from purified $30 \mathrm{~S}$ subunits from $\triangle$ rim $M$ and parental cells, respectively. Labeling reactions from both samples were combined and dried by lyophiliza- tion. iTRAQ-labeled peptides underwent NanoLC using a Dionex Ultimate 3000 . Tryptic peptides were loaded onto a $\mu$-Precolumn Cartridge and separated on an acetonitrile gradient (ranging from $5 \%$ to $60 \%$ ) on a Nano LC column. Fractions were collected at 20sec intervals, followed by mass spectrometry analysis on AB SCIEX TOF/TOF 5800 System (AB SCIEX). Mass spectra were acquired in positive ion reflection mode. TOF/TOF tandem MS fragmentation spectra were acquired for each ion, averaging 4000 laser shots per fragmentation spectrum (excluding trypsin autolytic peptides and other known background ions).

The resulting fragmentation spectra were submitted to GPS Explorer workstation equipped with MASCOT search engine (Matrix Science) to search the database of National Center for Biotechnology Information nonredundant (NCBInr). Searches were performed without constraining protein molecular weight or isoelectric point, with variable methylthiolation of cysteine and oxidation of methionine residues, fixed $\mathrm{N}$-terminal- and lysine-modifications with iTRAQ labels, and one missed cleavage. Quantitation was performed on peptides displaying an ion score confidence interval of $99 \%$ or higher.

\section{Cryo-electron microscopy and image analysis}

Holey carbon grids with an additional continuous layer of thin carbon $(5-10 \mathrm{~nm})$ were glow discharged at $5 \mathrm{~mA}$ for $15 \mathrm{sec}$ immediately before a $3.4 \mu \mathrm{L}$ drop of sample was deposited on the grid. Samples contained purified $30 \mathrm{~S}$ subunits from $\triangle$ rimM cells in buffer $\mathrm{E}$ at a concentration of $60 \mathrm{nM}$. We used a Vitrobot (FEI) to blot the grids and perform the vitrification of the specimen in liquid ethane. The blotting chamber of the Vitrobot was set up at $25^{\circ} \mathrm{C}$ and $100 \%$ relative humidity. Grids were blotted twice, 7 sec each time, before they were plunged into liquid ethane. Grids were transferred to a JEOL 2010F electron microscope operated at $200 \mathrm{kV}$ using a Gatan 914 cryo-holder.

Electron micrographs were collected under low dose conditions $\left(10-15 \mathrm{e}^{-} / \AA^{2}\right)$ at a nominal magnification of 50,000 $\times$. Electron micrographs were collected using a range of defocus from -1.5 to -4 $\mu \mathrm{m}$ and digitalized with a step size of $12.7 \mu \mathrm{m}$ in a Nikon Supercool Scan 9000 . The sampling of the images was $2.54 \AA$ /pixel.

A total of 86,205 particles representing the 30 S subunits purified from $\triangle$ rim $M$ cells were selected from the micrographs using Boxer (Ludtke et al. 1999). CTF parameter estimation was done using CTFFIND (Mindell and Grigorieff 2003), and the CTF correction was performed using Xmipp (Scheres et al. 2008).

The $2 \mathrm{D}$ analysis of the projection images was performed using a reference-free maximum likelihood-based classification approach as implemented in the Xmipp (Scheres et al. 2008). The classification was run for a total of 100 iterations.

The supervised classification to separate contaminating projections of mature $30 \mathrm{~S}$ subunits was performed as previously described (Jomaa et al. 2011a) using Spider (Valle et al. 2002; Gao et al. 2004). The three subpopulations of particles resulting from the classification were refined independently using the projection matching approach as implemented in the Xmipp program (Scheres et al. 2008). The initial maps for these refinements were produced using a maximum likelihood-based approach (Scheres et al. 2005b,c) to prevent model bias.

Classifications using a maximum likelihood-based approach were done as previously described (Scheres et al. 2005a,c, 2007). Briefly, 
the cryo-EM structure of the mature E. coli 30 S subunit (Jomaa et al. 2011a) (EMDB ID 1775) low-pass filtered to $70 \AA$ was used as an initial reference to produce four seeds from random subsets of experimental images by performing one iteration of maximum likelihood optimization. We obtained four homogeneous subpopulations after 25 iterations of maximum likelihood-based classification with these four seeds and twofold downscaled images of the data set. Each subpopulation of particles was referred back to the full size images to produce a $3 \mathrm{D}$ map using projection matching approaches. The reference maps for the projection matching refinements were produced from the particles in each subclass using a maximum likelihood-based refinement approach (Scheres et al. 2005b,c) to prevent model bias. Estimation of the resolution of the final maps was done from the even- and odd-numbered particles following the last cycle of refinement. Each group of particles produced a 3D map, and from the two maps, we obtained the Fourier shell correlation. Resolution value was obtained from this plot using the 0.5 criteria, and this value was used to low-pass filter the final three-dimensional reconstructions (Supplemental Fig. S1).

The Chimera program (Pettersen et al. 2004) was used to visualize the cryo-EM maps and to also perform the rigid body fitting of the X-ray structure of the E. coli $30 \mathrm{~S}$ subunit (PDB ID: 2AVY) (Schuwirth et al. 2005) into the 3D density maps.

\section{DATA DEPOSITION}

The cryo-EM maps reported here have been deposited in the Electron Microscopy Data Bank. The EMDB IDs are 5595, 5596, 5597, and 5598 for the conformational subpopulations CL 1, CL 2, CL 3, and CL 4 in Figure 3, respectively.

\section{SUPPLEMENTAL MATERIAL}

Supplemental material is available for this article.

\section{ACKNOWLEDGMENTS}

We thank the staff at the EM Facility of the Faculty of Sciences and at the Canadian Centre for Electron Microscopy at McMaster. This work is supported by a grant from the National Science and Engineering Research Council of Canada (RGPIN288327-07) to J.O. A.J. is supported by a CIHR Doctoral Research Award. We thank Dr. Eric Brown for helpful discussions on the manuscript and making accessible clones from the Keio and ASKA collection. The funders had no role in study design, data collection and analysis, decision to publish, or preparation of the manuscript.

Received November 30, 2012; accepted March 12, 2013.

\section{REFERENCES}

Adilakshmi T, Bellur DL, Woodson SA. 2008. Concurrent nucleation of $16 \mathrm{~S}$ folding and induced fit in $30 \mathrm{~S}$ ribosome assembly. Nature 455: $1268-1272$.

Anantharaman V, Aravind L. 2002. The PRC-barrel: A widespread, conserved domain shared by photosynthetic reaction center subunits and proteins of RNA metabolism. Genome Biol 3: RESEARCH0061.

Baba T, Ara T, Hasegawa M, Takai Y, Okumura Y, Baba M, Datsenko KA, Tomita M, Wanner BL, Mori H. 2006. Construction of Escherichia coli K-12 in-frame, single-gene knockout mutants: The Keio collection. Mol Syst Biol 2: 2006.0008. doi: 10.1038/msb4100050.

Britton RA. 2009. Role of GTPases in bacterial ribosome assembly. Annu Rev Microbiol 63: 155-176.

Brow DA, Noller HF. 1983. Protection of ribosomal RNA from kethoxal in polyribosomes. Implication of specific sites in ribosome function. J Mol Biol 163: 27-46.

Bunner AE, Trauger SA, Siuzdak G, Williamson JR. 2008. Quantitative ESI-TOF analysis of macromolecular assembly kinetics. Anal Chem 80: 9379-9386.

Bylund GO, Persson BC, Lundberg LA, Wikstrom PM. 1997. A novel ribosome-associated protein is important for efficient translation in Escherichia coli. J Bacteriol 179: 4567-4574.

Bylund GO, Wipemo LC, Lundberg LA, Wikstrom PM. 1998. RimM and RbfA are essential for efficient processing of $16 \mathrm{~S}$ rRNA in Escherichia coli. J Bacteriol 180: 73-82.

Bylund GO, Lovgren JM, Wikstrom PM. 2001. Characterization of mutations in the metY-nusA-infB operon that suppress the slow growth of a $\Delta$ rimM mutant. J Bacteriol 183: 6095-6106.

Campbell TL, Brown ED. 2008. Genetic interaction screens with ordered overexpression and deletion clone sets implicate the Escherichia coli GTPase YjeQ in late ribosome biogenesis. J Bacteriol 190: 2537-2545.

Campbell TL, Henderson J, Heinrichs DE, Brown ED. 2006. The yjeQ gene is required for virulence of Staphylococcus aureus. Infect Immun 74: 4918-4921.

Connolly K, Culver G. 2009. Deconstructing ribosome construction. Trends Biochem Sci 34: 256-263.

Daigle DM, Rossi L, Berghuis AM, Aravind L, Koonin EV, Brown ED. 2002. YjeQ, an essential, conserved, uncharacterized protein from Escherichia coli, is an unusual GTPase with circularly permuted G-motifs and marked burst kinetics. Biochemistry 41: 11109-11117.

Datta PP, Wilson DN, Kawazoe M, Swami NK, Kaminishi T, Sharma MR, Booth TM, Takemoto C, Fucini P, Yokoyama S, et al. 2007. Structural aspects of RbfA action during small ribosomal subunit assembly. Mol Cell 28: 434-445.

Gao H, Valle M, Ehrenberg M, Frank J. 2004. Dynamics of EF-G interaction with the ribosome explored by classification of a heterogeneous cryo-EM dataset. J Struct Biol 147: 283-290.

Goto S, Kato S, Kimura T, Muto A, Himeno H. 2011. RsgA releases RbfA from $30 \mathrm{~S}$ ribosome during a late stage of ribosome biosynthesis. EMBO J 30: 104-114.

Grishin NV. 2001. KH domain: One motif, two folds. Nucleic Acids Res 29: 638-643.

Guo Q, Yuan Y, Xu Y, Feng B, Liu L, Chen K, Sun M, Yang Z, Lei J, Gao N. 2011. Structural basis for the function of a small GTPase RsgA on the 30S ribosomal subunit maturation revealed by cryoelectron microscopy. Proc Natl Acad Sci 108: 13100-13105.

Hayes F, Hayes DH. 1971. Biosynthesis of ribosomes in E. coli. I. Properties of ribosomal precursor particles and their RNA components. Biochimie 53: 369-382.

Himeno H, Hanawa-Suetsugu K, Kimura T, Takagi K, Sugiyama W, Shirata S, Mikami T, Odagiri F, Osanai Y, Watanabe D, et al. 2004. A novel GTPase activated by the small subunit of ribosome. Nucleic Acids Res 32: 5303-5309.

Inoue K, Alsina J, Chen J, Inouye M. 2003. Suppression of defective ribosome assembly in a rbfA deletion mutant by overexpression of Era, an essential GTPase in Escherichia coli. Mol Microbiol 48: 1005-1016.

Inoue K, Chen J, Tan Q, Inouye M. 2006. Era and RbfA have overlapping function in ribosome biogenesis in Escherichia coli. J Mol Microbiol Biotechnol 11: 41-52.

Jiang M, Datta K, Walker A, Strahler J, Bagamasbad P, Andrews PC, Maddock JR. 2006. The Escherichia coli GTPase CgtA $A_{E}$ is involved in late steps of large ribosome assembly. J Bacteriol 188: $6757-6770$.

Jomaa A, Stewart G, Martin-Benito J, Zielke R, Campbell TL, Maddock JR, Brown ED, Ortega J. 2011a. Understanding ribosome 
assembly: The structure of in vivo assembled immature $30 \mathrm{~S}$ subunits revealed by cryo-electron microscopy. RNA 17: 697-709.

Jomaa A, Stewart G, Mears JA, Kireeva I, Brown ED, Ortega J. 2011 b. Cryo-electron microscopy structure of the $30 \mathrm{~S}$ subunit in complex with the YjeQ biogenesis factor. RNA 17: 2026-2038.

Kitagawa M, Ara T, Arifuzzaman M, Ioka-Nakamichi T, Inamoto E, Toyonaga H, Mori H. 2005. Complete set of ORF clones of Escherichia coli ASKA library (a complete set of E. coli K-12 ORF archive): Unique resources for biological research. DNA Res 12: 291-299.

Li Z, Pandit S, Deutscher MP. 1999. RNase G (CafA protein) and RNase $\mathrm{E}$ are both required for the $5^{\prime}$ maturation of $16 \mathrm{~S}$ ribosomal RNA. EMBO J 18: 2878-2885.

Lindahl L. 1975. Intermediates and time kinetics of the in vivo assembly of Escherichia coli ribosomes. J Mol Biol 92: 15-37.

Lovgren JM, Wikstrom PM. 2001. Hybrid protein between ribosomal protein S16 and RimM of Escherichia coli retains the ribosome maturation function of both proteins. J Bacteriol 183: 53525357.

Lovgren JM, Bylund GO, Srivastava MK, Lundberg LA, Persson OP, Wingsle G, Wikstrom PM. 2004. The PRC-barrel domain of the ribosome maturation protein RimM mediates binding to ribosomal protein S19 in the 30 S ribosomal subunits. RNA 10: 17981812.

Ludtke SJ, Baldwin PR, Chiu W. 1999. EMAN: Semiautomated software for high-resolution single-particle reconstructions. J Struct Biol 128: 82-97.

Mindell JA, Grigorieff N. 2003. Accurate determination of local defocus and specimen tilt in electron microscopy. J Struct Biol 142: 334-347.

Nierhaus KH. 1980. The assembly of the prokaryotic ribosome. Biosystems 12: 273-282.

Nord S, Bylund GO, Lovgren JM, Wikstrom PM. 2009. The RimP protein is important for maturation of the $30 \mathrm{~S}$ ribosomal subunit. J Mol Biol 386: 742-753.

Pettersen EF, Goddard TD, Huang CC, Couch GS, Greenblatt DM, Meng EC, Ferrin TE. 2004. UCSF Chimera-a visualization system for exploratory research and analysis. J Comput Chem 25: 1605-1612.

Potrykus K, Cashel M. 2008. (p)ppGpp: Still magical? Annu Rev Microbiol 62: 35-51.

Ron EZ, Kohler RE, Davis BD. 1966. Polysomes extracted from Escherichia coli by freeze-thaw-lysozyme lysis. Science 153: 1119-1120.

Ross PL, Huang YN, Marchese JN, Williamson B, Parker K, Hattan S, Khainovski N, Pillai S, Dey S, Daniels S, et al. 2004. Multiplexed protein quantitation in Saccharomyces cerevisiae using amine-reactive isobaric tagging reagents. Mol Cell Proteomics 3: 1154-1169.

Sambrook J, Fritsch EF, Maniatis T. 1989. Molecular cloning: A laboratory manual. Cold Spring Harbor Laboratory Press, Cold Spring Harbor, NY.
Scheres SH, Marabini R, Lanzavecchia S, Cantele F, Rutten T, Fuller SD, Carazo JM, Burnett RM, San Martin C. 2005a. Classification of single-projection reconstructions for cryo-electron microscopy data of icosahedral viruses. J Struct Biol 151: 79-91.

Scheres SH, Valle M, Carazo JM. 2005b. Fast maximum-likelihood refinement of electron microscopy images. Bioinformatics 21 (Suppl 2): i243-ii244.

Scheres SH, Valle M, Nunez R, Sorzano CO, Marabini R, Herman GT, Carazo JM. 2005c. Maximum-likelihood multi-reference refinement for electron microscopy images. J Mol Biol 348: 139-149.

Scheres SH, Gao H, Valle M, Herman GT, Eggermont PP, Frank J, Carazo JM. 2007. Disentangling conformational states of macromolecules in 3D-EM through likelihood optimization. Nat Methods 4: 27-29.

Scheres SH, Nunez-Ramirez R, Sorzano CO, Carazo JM, Marabini R. 2008. Image processing for electron microscopy single-particle analysis using XMIPP. Nat Protoc 3: 977-990.

Schuwirth BS, Borovinskaya MA, Hau CW, Zhang W, Vila-Sanjurjo A, Holton JM, Cate JH. 2005. Structures of the bacterial ribosome at 3.5 Å resolution. Science 310: 827-834.

Shajani Z, Sykes MT, Williamson JR. 2011. Assembly of bacterial ribosomes. Annu Rev Biochem 80: 501-526.

Sharma MR, Barat C, Wilson DN, Booth TM, Kawazoe M, HoriTakemoto C, Shirouzu M, Yokoyama S, Fucini P, Agrawal RK. 2005. Interaction of Era with the 30 S ribosomal subunit implications for 30S subunit assembly. Mol Cell 18: 319-329.

Sieber G, Nierhaus KH. 1978. Kinetic and thermodynamic parameters of the assembly in vitro of the large subunit from Escherichia coli ribosomes. Biochemistry 17: 3505-3511.

Srivastava AK, Schlessinger D. 1990. Mechanism and regulation of bacterial ribosomal RNA processing. Annu Rev Microbiol 44: 105-129.

Suzuki S, Tatsuguchi A, Matsumoto E, Kawazoe M, Kaminishi T, Shirouzu M, Muto Y, Takemoto C, Yokoyama S. 2007. Structural characterization of the ribosome maturation protein, RimM. $J$ Bacteriol 189: 6397-6406.

Sykes MT, Shajani Z, Sperling E, Beck AH, Williamson JR. 2010. Quantitative proteomic analysis of ribosome assembly and turnover in vivo. J Mol Biol 403: 331-345.

Talkington MW, Siuzdak G, Williamson JR. 2005. An assembly landscape for the 30S ribosomal subunit. Nature 438: 628-632.

Valle M, Sengupta J, Swami NK, Grassucci RA, Burkhardt N, Nierhaus KH, Agrawal RK, Frank J. 2002. Cryo-EM reveals an active role for aminoacyl-tRNA in the accommodation process. EMBO J 21: $3557-3567$.

Williamson JR. 2005. Assembly of the 30 S ribosomal subunit. Q Rev Biophys 38: 397-403.

Wilson DN, Nierhaus KH. 2007. The weird and wonderful world of bacterial ribosome regulation. Crit Rev Biochem Mol Biol 42: 187-219. 

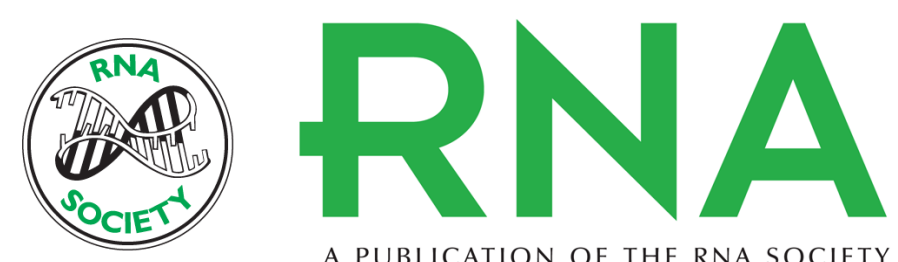

A PUBLICATION OF THE RNA SOCIETY

\section{Escherichia coli rimM and yjeQ null strains accumulate immature $30 \mathrm{~S}$ subunits of similar structure and protein complement}

Vivian Leong, Meredith Kent, Ahmad Jomaa, et al.

RNA 2013 19: 789-802 originally published online April 23, 2013

Access the most recent version at doi:10.1261/rna.037523.112

\section{Supplemental http://rnajournal.cshlp.org/content/suppl/2013/04/08/rna.037523.112.DC1 \\ Material}

References This article cites 54 articles, 18 of which can be accessed free at:

http://rnajournal.cshlp.org/content/19/6/789.full.html\#ref-list-1

\section{License}

Email Alerting Receive free email alerts when new articles cite this article - sign up in the box at the Service top right corner of the article or click here.

\section{||I|||| Providing Precise Solutions for your research.}

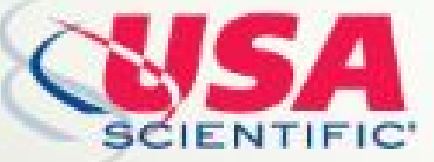

To subscribe to $R N A$ go to:

http://rnajournal.cshlp.org/subscriptions 